\title{
RESEARCH
}

Open Access

\section{Ceria nanoparticles ameliorate white matter injury after intracerebral hemorrhage: microglia-astrocyte involvement in remyelination}

Jingwei Zheng ${ }^{1}$, Jia'nan Lu', Shuhao Mei', Haijian Wu' ${ }^{1}$ Zeyu Sun ${ }^{1}$, Yuanjian Fang ${ }^{1}$, Shenbin Xu', Xiaoyu Wang ${ }^{1}$, Ligen Shi ${ }^{1}$, Weilin $\mathrm{Xu}^{1}$, Sheng Chen ${ }^{1}$, Jun $\mathrm{Yu}^{{ }^{1 *}}{ }^{\mathbb{D}}$, Feng Liang ${ }^{1 *}$ and Jianmin Zhang ${ }^{1,2,3^{*}}$

\begin{abstract}
Background: Intracerebral hemorrhage $(\mathrm{ICH})$ can induce excessive accumulation of reactive oxygen species (ROS) that may subsequently cause severe white matter injury. The process of oligodendrocyte progenitor cell (OPC) differentiation is orchestrated by microglia and astrocytes, and ROS also drives the activation of microglia and astrocytes. In light of the potent ROS scavenging capacity of ceria nanoparticles (CeNP), we aimed to investigate whether treatment with CeNP ameliorates white matter injury by modulating ROS-induced microglial polarization and astrocyte alteration.
\end{abstract}

Methods: ICH was induced in vivo by collagenase VII injection. Mice were administered with PLX3397 for depleting microglia. Primary microglia and astrocytes were used for in vitro experiments. Transmission electron microscopy analysis and immunostaining were performed to verify the positive effects of CeNP in remyelination and OPC differentiation. Flow cytometry, real-time polymerase chain reaction, immunofluorescence and western blotting were used to detect microglia polarization, astrocyte alteration, and the underlying molecular mechanisms.

Results: CeNP treatment strongly inhibited ROS-induced NF-KB p65 translocation in both microglia and astrocytes, and significantly decreased the expression of $\mathrm{M} 1$ microglia and $\mathrm{A} 1$ astrocyte. Furthermore, we found that CeNP treatment promoted remyelination and OPC differentiation after $\mathrm{ICH}$, and such effects were alleviated after microglial depletion. Interestingly, we also found that the number of mature oligodendrocytes was moderately increased in ICH + CeNP + PLX3397-treated mice compared to the ICH + vehicle + PLX3397 group. Therefore, astrocytes might participate in the pathophysiological process. The subsequent phagocytosis assay indicated that A1 astrocyte highly expressed C3, which could bind with microglia C3aR and hinder microglial engulfment of myelin debris. This result further replenished the feedback mechanism from astrocytes to microglia.

(Continued on next page)

\footnotetext{
* Correspondence: 2505020@zju.edu.cn; frankfeng@zju.edu.cn; zjm135@zju.edu.cn

'Department of Neurosurgery, Second Affiliated Hospital, School of Medicine, Zhejiang University, Hangzhou, Zhejiang, China

Full list of author information is available at the end of the article
} 


\begin{abstract}
(Continued from previous page)
Conclusion: The present study reveals a new mechanism in white matter injury after $\mathrm{ICH}$ : ICH induces $\mathrm{M} 1$ microglia and A1 astrocyte through ROS-induced NF-KB p65 translocation that hinders OPC maturation. Subsequently, A1 astrocytes inhibit microglial phagocytosis of myelin debris via an astrocytic C3-microglial C3aR axis. Polyethylene glycol-CeNP treatment inhibits this pathological process and ultimately promotes remyelination. Such findings enlighten us that astrocytes and microglia should be regarded as a functional unit in future works.
\end{abstract}

Keywords: Intracerebral hemorrhage, Reactive oxygen species, White matter injury, Microglia, Astrocyte

\section{Introduction}

Intracerebral hemorrhage $(\mathrm{ICH})$ is a serious neurological crisis that accounts for $20-30 \%$ of stroke cases in Asia, with high mortality and disability $[1,2]$. White matter accounts for $50-60 \%$ of the human brain volume $[3,4]$. A previous study demonstrated that $77 \%$ of ICH patients had white matter injury (WMI) [5]. ICH commonly occurs around the basal ganglia, which contains abundant white matter fibers (internal capsule). Therefore, ICH can induce severe WMI in patients resulting in hemiplegia, hemianopsia, and sensory deficit.

In normal brain tissue, axons are enwrapped by the myelin sheath, which is associated with faster potential propagation and neurological functions [3, 6]. Oligodendrocytes (OLs) are responsible for myelination in the central nervous system (CNS), and individual mature OLs can myelinate up to 60 axons [7]. Previous studies have demonstrated that $\mathrm{ICH}$ induced severe oligodendrocyte death and demyelination in white matter [8]. OPCs play a vital role in remyelination as the damaged mature OLs are unable to proliferate and form new myelin sheaths. Although OPCs and immature OLs cannot myelinate axons immediately, OPCs become highly proliferative and then differentiate into mature OLs, which can remyelinate injured axons after demyelinating injuries $[9,10]$. The process of OPC proliferation and differentiation is orchestrated by microglia and astrocytes. Microglia are the foremost and earliest immune cells that participate in the pathological process after $\mathrm{ICH}[2$, 11]. In response to microenvironmental cues, microglia may exhibit different polarization states ranging from neurotoxic to protective phenotypes, including M1 and M2 polarized status. Oligodendrocyte proliferation and differentiation can be promoted by M2 antiinflammatory microglia and impaired by M1 proinflammatory microglia $[11,12]$. Notably, astrocytes and microglia have a unique bond, and both always respond as one unit when the brain is perturbed [13]. A previous study demonstrated that several CNS diseases resulted in reactive astrocytes, which could be transformed into the A1 phenotype via mediators (IL-1 $\alpha, \mathrm{C} 1 \mathrm{q}, \mathrm{TNF}$ ) secreted by activated microglia [14]. The A1 neurotoxic astrocytes can further induce the death of neurons and oligodendrocytes and inhibit OPC maturation.
Therefore, there appears to be a strong relationship between microglia-astrocytes crosstalk and remyelination.

Reactive oxygen species (ROS) are excessively produced in perihematomal white matter after $\mathrm{ICH}$, which can oxidize protein, lipids, and nuclear material [15]. Oligodendrocyte-lineage cells (including OPCs and OLs) are rich in lipids and lack essential glutathione and glutathione peroxidase. These specific characteristics make it highly vulnerable to oxidative stress, which ultimately results in WMI [16]. Notably, ROS can induce microglia activation and perturb the kinetics of the M1/ M2 shift [17-19]. The scavenging of microglial ROS through genetic abrogation of NADPH oxidase attenuates the pro-inflammatory M1 response and elevates the anti-inflammatory M2 markers [19]. Except for the inflammatory stimuli, ROS have also been implicated in driving astrocyte reactivity $[20,21]$. Therefore, ROS scavenging may be a promising way to modulate the activation of microglia and astrocyte and subsequently promote remyelination.

Ceria nanoparticle (CeNP) is known to possess a potent-free radical scavenging activity. The high pseudoenzyme activity enables $1.0 \mu \mathrm{g}$ of CeNP to exhibit an equivalent ability to neutralize ROS to $527 \mathrm{U}$ of superoxide dismutase (SOD) [22]. Particle miniaturization results in the generation of oxygen vacancies, which leads to the co-existence of $\mathrm{Ce}^{+}$and $\mathrm{Ce}^{+}$on the crystalline lattice $[18,23]$. As a result, CeNP has SOD mimetic $\left(\mathrm{Ce}^{+}\right)$ and catalase mimetic $\left(\mathrm{Ce}^{+}\right)$together, which enables CeNP to repetitively eradicate ROS [23]. Here, we investigated the putative therapeutic effects of CeNP on WMI via its ROS scavenging capacity, focusing on the role of microglia and astrocytes in remyelination after ICH.

\section{Method}

\section{Ethics statement}

All animal procedures were carried out in accordance with the Guide for the Care and Use of Laboratory Animals of the National Institutes of Health and were approved by the Institutional Animal Care and Use Committee of Zhejiang University. The animals were raised 3 per cage with controlled temperature and humidity and a 12-h light/dark cycle. 


\section{Experimental animals}

Adult male C57BL/ 6 wild type (WT) mice (20-25 g) and mixed-sex C57BL/6 WT mice pups (postnatal within 24 h) were purchased from Slac Laboratory Co., Ltd. (Shanghai, China).

\section{Administration of PLX3397}

The survival of microglia depends on colony-stimulating factor 1 receptor (CSF1R) signaling. Reportedly, dietary treatment with a CSF1R inhibitor PLX3397 effectively depletes microglia in mice without apparent abnormalities in neurological function [24]. For microglia/macrophage depletion, PLX3397 (Selleck chem, Houston, TX) was formulated in AIN-76A standard chow at $290 \mathrm{mg} /$ $\mathrm{kg}$, starting 21 days before $\mathrm{ICH}$ surgery until the end of the experiments [24].

\section{$\mathrm{ICH}$ induction}

C57BL/6 mice were anesthetized by injecting pentobarbital $(40 \mathrm{mg} / \mathrm{kg})$ intraperitoneally and placed in a stereotaxic frame (Stoelting Stereotaxic instrument). A 1-mm burr hole in diameter was made in the skull $(0.2 \mathrm{~mm}$ anterior to the bregma, $2.2 \mathrm{~mm}$ right lateral to the midline). Then, the experimental ICH model was induced by injecting bacterial collagenase VII $(0.1 \mathrm{U}$ in $0.5 \mu \mathrm{L}$, Sigma, St. Louis, MO) into the right basal ganglia (3.5 $\mathrm{mm}$ depth below the skull) using a micro-perfusion pump within $5 \mathrm{~min}$. In case backflows occurred, the needle was not removed for an additional $10 \mathrm{~min}$ after the completion of the injection [2, 25]. The burr hole was blocked with bone wax. In the sham group, C57BL/6 mice underwent the same procedures, but $0.5 \mu \mathrm{L}$ saline was injected instead of collagenase. During the procedure, rectal temperature was maintained at $37.0 \pm 0.5{ }^{\circ} \mathrm{C}$ using a temperature-regulated heating pad.

\section{Synthesis of polyethylene glycol (PEG)-CeNPs and administration}

As described previously [18], mPEG2000-DSPE $30 \mathrm{mg}$, $1.1 \times 10^{-2} \mathrm{mmol}$, was dissolved in $1.0 \mathrm{~mL}$ of chloroform. Then it was mixed with $10 \mathrm{mg}$ CeNPs $(<5 \mathrm{~nm}$, Sigma, St. Louis, MO) and stirred for $4 \mathrm{~h}$ at room temperature. After removing the chloroform, $10 \mathrm{~mL}$ of ultra-pure water was added, and the mixture was dispersed by stirring at $2000 \mathrm{rpm}$ for $2 \mathrm{~h}$, bath sonication for $30 \mathrm{~min}$ at $30 \%$ amplitude, and full cycle. Finally, the colloidal stabilities of PEG-CeNPs were evaluated by observing their clarity after storage in pure water at $4{ }^{\circ} \mathrm{C}$. The hydrodynamic size distribution and zeta potential of CeNPPEG in DI water were measured by dynamic light scattering (DLS).

Then, CeNPs ( $0.5 \mathrm{mg} / \mathrm{kg}$ body weight) or an equal volume of phosphate buffer saline (PBS) was intravenously injected at $6 \mathrm{~h}$ and $30 \mathrm{~h}$ after $\mathrm{ICH}$ and then repeated every 3 days

\section{Dihydroethidium (DHE) injection}

DHE was injected intraperitoneally to label superoxide in vivo. At 3 days post $\mathrm{ICH}$, the mice were intraperitoneally injected with DHE $(0.01 \mathrm{mg} / \mathrm{g}$ body weight). Then, the mice were euthanized $4 \mathrm{~h}$ after injection [26]. Afterward, the coronal cryosections were observed using a fluorescence microscope.

\section{ROS assay}

In the brain tissue, ROS production was tested using a ROS assay kit (JianCheng, Nanjing, China) according to the manufacturer's instructions. Mice were euthanized at 3 days post $\mathrm{ICH}$. The brain tissue samples were lysed in $0.01 \mathrm{~mol} / \mathrm{L} \mathrm{PBS}$ and centrifuged at $4{ }^{\circ} \mathrm{C} 500 \times g$ for 10 min. Then, the supernatant $(190 \mu \mathrm{l})$ and dichlorodihydro-fluorescein diacetate (DCFH-DA; $10 \mu \mathrm{l}, 1 \mathrm{~mol} / \mathrm{L}$ ) were mixed in a micro-well at room temperature for 30 min [2]. Then, the protein levels of the different samples were acquired using a detergent-compatible protein assay kit (Bio-Rad, Hercules, CA, USA). Ultimately, the ROS levels were displayed in the form of fluorescence/ mg protein. Likewise, the microglia or astrocyte suspensions were incubated with $10 \mu \mathrm{M}$ DCFH-DA in 96-well plates for $30 \mathrm{~min}$ at room temperature. Afterward, these mixtures were measured with a fluorophotometer.

\section{Transmission electron microscopy (TEM)}

As described previously [3], TEM was used to measure myelin thickness in the striatum area. Three mice per group were euthanized at 21 days after $\mathrm{ICH}$ and then perfused with $0.9 \%$ saline and $2.5 \%$ glutaraldehyde. Furthermore, $1-\mathrm{mm}^{3}$ fragments of peri-hematoma tissue were obtained from the right basal ganglia and then processed with glutaraldehyde $(2.5 \%)$ at $4{ }^{\circ} \mathrm{C}$ overnight. Then, the tissues were further handled through a succession of chemical treatment steps (1\% osmium tetroxide, and distilled water). Afterward, the samples were embedded in a mixture of propylene oxide and resin (1:1) overnight and were sliced into $60-\mathrm{nm}$ sections and then stained with $4 \%$ uranyl acetate and $0.5 \%$ lead citrate. The ultrastructure of the myelin was scanned using TEM (Philips Tecnai 10). The electron micrographs were taken at $15 \mathrm{~K}$ magnification, and Image J software was used to measure the myelin thickness. G-ratios were calculated as the ratio of the inner axonal diameter to the total outer diameter $(\mathrm{d} / \mathrm{D})$.

\section{Flow cytometry}

Mice were euthanized and perfused with cold PBS. As described previously [2], the brain tissue was dissociated mechanically and digested in $6 \mathrm{ml}$ of DMEM medium 
with $0.6 \mathrm{mg} / \mathrm{mL}$ collagenase IV (Worthington) for 30 $\min$ at $37^{\circ} \mathrm{C}$. Then, the suspension was filtered with a $70-\mu \mathrm{m}$ cell strainer (BD Biosciences), and the cells were resuspended in $70 \%$ Percoll. Then, the cell suspension was separated from the myelin and debris after centrifugation $\left(700 \mathrm{~g}, 4{ }^{\circ} \mathrm{C}, 40 \mathrm{~min}\right)$ on a $37-70 \%$ Percoll gradient. The inflammatory cells were carefully collected from the interface of the gradient and washed with 10 $\mathrm{ml}$ of PBS. Single-cell samples were incubated with CD45-percp (1:200, BD PharMingen), CD11b-FITC (1: 200, BD PharMingen), CD16/32-PE (1:200, BDPharMingen), and CD206-APC (1:200, BD PharMingen) at $4{ }^{\circ} \mathrm{C}$ in the dark for $25 \mathrm{~min}$. CD $45^{+} \mathrm{CD} 11 \mathrm{~b}^{+}$cells were considered as microglia $\left(\mathrm{CD} 45^{+ \text {low }} \mathrm{CD} 11 \mathrm{~b}^{+}\right)$and macrophages $\left(\mathrm{CD} 45^{\text {thigh }} \mathrm{CD} 11 \mathrm{~b}^{+}\right) . \mathrm{CD} 16 / 32^{+}$and $\mathrm{CD} 206^{+}$cells were acknowledged as M1 and M2 phenotype microglia, respectively. The subsequent flow cytometric analysis was performed using a FACS flow cytometer C6 (BD Biosciences), and the results were analyzed using FlowJo version 7.6.1.

\section{Primary microglia and astrocyte culture and treatment} Cortices were isolated from newborn pups (within $24 \mathrm{~h}$ ) after removing the meninges and blood vessels in Hank's balanced salt solution and cut into small pieces. The tissue was digested with $2.5 \%$ trypsin at $37^{\circ} \mathrm{C}$ for $15 \mathrm{~min}$ before DMEM was added. Then the single-cell suspension was filtered with a $70-\mu \mathrm{m}$ cell strainer. After centrifugation and resuspension, mixed glial cells were plated onto poly-D-lysine-coated (PDL) T-25 flasks and cultured with DMEM medium containing $10 \%$ fetal bovine serum. These cells were incubated at $37^{\circ} \mathrm{C}$ in a humidified $5 \% \mathrm{CO}_{2}, 95 \%$ air atmosphere. The cell culture medium was changed $24 \mathrm{~h}$ after plating, then renewed every 3 days. Then, 7 to 10 days later, microglia were separated from mixed glial cells by orbital shaking at $180 \mathrm{rpm}$ for $1 \mathrm{~h}$. Afterward, the supernatant containing microglia was obtained and re-plated on PDL-coated Tflasks. For the astrocyte culture, the flask with mixed glial cultures was shaken for $3 \mathrm{~h}$ at $250 \mathrm{rpm}$ to remove unwanted cells including OPCs, microglia, neurons and fibroblasts. Then the astrocytes were digested with $2.5 \%$ trypsin at $37^{\circ} \mathrm{C}$ for $5 \mathrm{~min}$ and re-plated on $\mathrm{T}-75$ flasks $[27,28]$.

Microglia were incubated with $\mathrm{H}_{2} \mathrm{O}_{2} 100 \mu \mathrm{M}$ for $24 \mathrm{~h}$. Astrocytes were incubated with $\mathrm{H}_{2} \mathrm{O}_{2} 100 \mu \mathrm{M}$ for $6 \mathrm{~h}$. In the treatment group, $0.32 \mu \mathrm{g} / \mathrm{mL}$ of PEG-CeNPs was incubated with microglia or astrocytes for $4 \mathrm{~h}$ and then washed twice with PBS before $\mathrm{H}_{2} \mathrm{O}_{2}$ treatment [18].

\section{Nissl staining}

Coronal cryosections were stained with Nissl staining solution (Beyotime, C0117) for $30 \mathrm{~min}$ at $37{ }^{\circ} \mathrm{C}$. Then, the cryosections were washed using 95\% ethyl alcohol and observed using an optical microscope. A large cell body, with abundant cytoplasm and substantially significant levels of Nissl body, represents a normal neuron. Cells with karyopyknosis or blurred Nissl bodies represented damaged ones.

\section{Immunofluorescence staining and image analysis}

The mice were euthanized under deep pentobarbital anesthesia at 3,7 , and 21 days after $\mathrm{ICH}$, and then perfused with $0.1 \mathrm{~mol} / \mathrm{L}$ PBS and $4 \%$ paraformaldehyde (PFA). The coronal cryosections and primary cultured cells (microglia and astrocytes) were preprocessed with $10 \%$ donkey serum and $0.3 \%$ Triton X-100, and then incubated at $4{ }^{\circ} \mathrm{C}$ with different antibodies overnight including MBP antibody (1:200, Santa Cruz, sc-66064), degraded myelin basic protein, and $\mathrm{dMBP}$ antibody (1: 400, Millipore, AB5864)

Anti-CD16/32 antibody (1:100, Abcam ab25235), antiCD206 antibody (1:500, Abcam ab64693), Iba-1 (1:500, Abcam ab5076), anti-NF- $\mathrm{kB}$ p65 antibody (1:500, CST \#6956), anti-olig2 antibody (1: 250, Millipore AB9610), anti-CC1 antibody (1: 500, Millipore OP80), anti-GFAP (Glial Fibrillary Acidic Protein) antibody (1:500, CST \#34001S), anti-Complement C3 antibody (1:400, Invitrogen PA5-21349), and anti-C3aR antibody (1:100, Hycult Biotech \#HM1123). Afterward, the cryosections or cultured cells were incubated with the secondary antibodies. Then, a fluorescence microscope (Olympus, Tokyo, Japan) was used to capture the images.

For MBP and AMBP staining, the areas of white matter lesion were assessed for each mouse brain. The areas of the MBP lesion or $\mathrm{AMBP}$ positive region were measured using ImageJ software. Likewise, positive staining cells were electronically counted using ImageJ software. Afterward, these data were calculated and analyzed.

\section{Phagocytosis assay}

Microglia were preprocessed with $10 \mu \mathrm{g} / \mathrm{ml}$ of C3 (Millipore; catalog no. \#204885) or A1 astrocyte conditioned medium (CM) for $24 \mathrm{~h}$. For the preparation of A1 astrocyte $\mathrm{CM}, \mathrm{A} 1$ astrocytes were maintained in basal medium without serum or growth factors for $24 \mathrm{~h}$. Collected conditioned medium were centrifuged $(2000 \mathrm{~g}, 10$ $\mathrm{min})$ to remove debris and then filtered through a 0.22 $\mu \mathrm{m}$ syringe filter. The A1 astrocyte $\mathrm{CM}$ was stored at $80^{\circ} \mathrm{C}$ before use. In the C3aR antagonist (C3aRA, SB290157, MCE HY-101502A) + A1 astrocyte CM group, $10 \mu \mathrm{M}$ of C3aRA was incubated with microglia for $1 \mathrm{~h}$ before the A1 astrocyte CM treatment. Then, aqueous green fluorescent beads $(1 \mu \mathrm{m}$ diameter, Sigma, \#L1030) were added into the medium for $3 \mathrm{~h}$. The final concentration $(\mathrm{v} / \mathrm{v})$ of beads and fetal bovine serum in the medium were $0.01 \%$ and $0.05 \%$, respectively [29]. 
Quantitative real-time polymerase chain reaction (RT-PCR) Total ribonucleic acid (RNA) was isolated from the mice brain tissue or primary cultured astrocyte using TRIzol reagent (Sigma-Aldrich, St. Louis, MO, USA), following the manufacturer's protocol. Then, cDNA synthesis was performed using a PrimeScriptTM RT reagent kit (Takara Bio Inc, Shiga, Japan). Afterward, cDNAs were amplified using SYBR ${ }^{\circ}$ Premix Ex Taq ${ }^{\mathrm{mm}}$ (Takara Bio Inc, Shiga, Japan) on a 7300 Plus Read-Time PCR System (Thermo Fisher Scientific). The PCR reaction was performed as follows. The cycling conditions began with an initial DNA denaturation step at $95^{\circ} \mathrm{C}$ for $20 \mathrm{~s}$, followed by 40 cycles at $94{ }^{\circ} \mathrm{C}$ for $15 \mathrm{~s}, 56{ }^{\circ} \mathrm{C}$ for $30 \mathrm{~s}$, and $72{ }^{\circ} \mathrm{C}$ for $25 \mathrm{~s}$. The cycle threshold values were collected and calculated with the $2^{-\Delta \Delta C T}$ method. Finally, mRNA expression was shown as fold changes versus sham controls. The target gene primers designed for quantitative RT-PCR are listed in Table 1.

\section{Western blotting}

Proteins were isolated from the mice brain tissue or cultured cells. Nuclear and cytoplasmic protein was separated using a Nuclear and Cytoplasmic Protein Extraction Kit (Beyotime, P0028). The cells or perihematoma tissues (basal ganglia) were homogenized in radio-immunoprecipitation assay lysis buffer (Beyotime). Then, the protein samples were separated by $10 \%$ or

Table 1 RT-PCR primer sequences

\begin{tabular}{|c|c|c|}
\hline ID & & Primer sequence \\
\hline \multirow[t]{2}{*}{ iNOS } & FWD & CAAGCACCTTGGAAGAGGAG \\
\hline & REV & AAGGCCAAACACAGCATACC \\
\hline \multirow[t]{2}{*}{ CD16 } & FWD & TITGGACACCCAGATGTTTCAG \\
\hline & REV & GTCTTCCTTGAGCACCTGGATC \\
\hline \multirow[t]{2}{*}{ IFN- $\gamma$} & FWD & CAGCAACAGCAAGGCGAAAAAGG \\
\hline & REV & TTTCCGCTTCCTGAGGCTGGAT \\
\hline \multirow[t]{2}{*}{ CD206 } & FWD & CAAGGAAGGTTGGCATTTGT \\
\hline & REV & CCTITCAGTCCTTTGCAAGC \\
\hline \multirow[t]{2}{*}{$Y M 1 / 2$} & FWD & CAGGGTAATGAGTGGGTTGG \\
\hline & REV & CACGGCACCTCCTAAATTGT \\
\hline \multirow[t]{2}{*}{$T G F-\beta$} & FWD & TGCGCTTGCAGAGATTAAAA \\
\hline & REV & CGTCAAAAGACAGCCACTCA \\
\hline \multirow[t]{2}{*}{ C3 } & FWD & CCAGCTCCCCATTAGCTCTG \\
\hline & REV & GCACTTGCCTCTITAGGAAGTC \\
\hline \multirow[t]{2}{*}{ Serping 1} & FWD & ACAGCCCCCTCTGAATTCTT \\
\hline & REV & GGATGCTCTCCAAGTTGCTC \\
\hline \multirow[t]{2}{*}{ Amigo2 } & FWD & GAGGCGACCATAATGTCGTT \\
\hline & REV & GCATCCAACAGTCCGATTCT \\
\hline \multirow[t]{2}{*}{ GAPDH } & FWD & AAGAGGGATGCTGCCCTTAC \\
\hline & REV & TACGGCCAAATCCGTTCACA \\
\hline
\end{tabular}

12\% SDS-PAGE and transferred onto polyvinylidene fluoride (PVDF) membranes (Millipore). Next, the polyvinylidene fluoride (PVDF) membranes were blocked with $5 \%$ bovine serum albumin for $1 \mathrm{~h}$ and incubated with the primary antibodies overnight, including antiphosphorylated NF- $\kappa B$ p65 antibody (1:500, Santa Cruz, sc-136548), anti-NF-kB p65 antibody (1:1000, CST \#6956), anti-IkB- $\alpha$ antibody (1:500, Santa Cruz, sc-1643), MBP antibody (1:500, Santa Cruz, sc-66064), Histone H3 (1:1000, CST \#9715) and $\beta$-actin (1:5000, Abcam, ab8226). After that, the PVDF membranes were disposed of with the relevant secondary antibodies (1:5000) for 1 $h$ at room temperature. The signals of the protein bands were detected using a Chemidoc detection system and quantified using Quantity One software (Bio-Rad).

\section{Behavioral tests \\ Accelerated rotarod test}

An accelerated rotarod test was performed using Rotarod Treadmills (BW-ZH600, Shanghai Bio-will Co., Ltd.) to test the motor coordination and limb strength of the mice. As described previously [25], the mice were placed on a six-lane accelerating rotarod (acceleration from 4 to $40 \mathrm{rpm}$ within $5 \mathrm{~min}$, increasing $4 \mathrm{rpm}$ every $30 \mathrm{~s}$ until reaching the final speed at $300 \mathrm{~s}$ ). The time during which the animals stayed on the rotarod was recorded. Each animal underwent this test 3 times a day. Data were expressed as the mean values from the 3 trials.

\section{Adhesive removal test}

As described previously $[2,3]$, the adhesive removal test was performed by an independent researcher to detect the tactile responses of the mice. Before the actual test, mice had been trained for 3 days to familiarize them with the test. Then, adhesive tape $(3 \mathrm{~mm} \times 3 \mathrm{~mm})$ was pasted on the left forepaw (affected side). The time to contact and remove the adhesive tape was measured (a maximum observation time $120 \mathrm{~s}$ ).

\section{Measurement of brain water content}

As described previously, the brain water content was measured using wet-dry method [2]; mice were euthanized under deep pentobarbital anesthesia at 3 days after $\mathrm{ICH}$. After removal of cerebellum and brain stem, the ipsilateral hemisphere was immediately weighed on an analytical balance (wet weight), and then dried at $100{ }^{\circ} \mathrm{C}$ for $24 \mathrm{~h}$ in the Electro-Thermostatic Blast Oven. The dry weight of these brain tissues was acquired by reweighting. Ultimately, brain water content was calculated using the following formula: [(wet weight-dry weight)/wet weight] $\times 100 \%$. 


\section{EdU injection}

In order to label proliferating cells, mice were intraperitoneally injected with a thymidine analog (5-ethynyl-2'deoxyuridine, EdU) at a dose of $50 \mathrm{mg} / \mathrm{kg}$ body weight. Mice were injected with EdU (Beyotime, Shanghai, China, C0075L) twice a day (with an interval of at least $8 \mathrm{~h}$ ) at 3 days after $\mathrm{ICH}$ for 4 consecutive days. Then EdU can be incorporated into cellular DNA and the subsequent reaction of EdU with a fluorescent azide in a copper-catalyzed [3+2] cycloaddition (Click reaction) $[3,30]$.

\section{Study design}

Animals were sacrificed as little as possible through rational experimental design, a total of 242 mice (including 30 neonatal mice) were used for this study at last. The detailed information is shown in Figure S1. The data collected was processed randomly and appropriately blocked. In addition, researchers were partly blind to group assignment and outcome assignment.

\section{Experiment 1}

In order to evaluate the effectiveness of PEG-CeNP, 12 mice were randomly (using random number table) divided into 2 groups: ICH $3 \mathrm{~d}$ + vehicle (PBS with PEG) $n$ $=6$, ICH 3 days + PEG-CeNP, $n=6$. Then, these animals were applied to Nissl staining. To observe relevant ROS levels, 12 mice were undergone DHE injection (ICH $3 \mathrm{~d}+$ vehicle $n=6$, ICH $3 \mathrm{~d}+$ PEG-CeNP, $n=6$ ), another 18 mice (Sham $n=6$, ICH 3 days + vehicle $n=$ 6 , ICH 3 days + PEG-CeNP, $n=6$ ) were used the quantify the ROS production by using a ROS assay kit. In total, 18 mice (Sham $n=6$, ICH 3 days + vehicle $n=6$, ICH 3 days + PEG-CeNP, $n=6$ ) were euthanized and used for brain water content test. Moreover, 9 mice (Sham $n=3$, ICH 21 days + vehicle $n=3$, ICH 21 days + PEG-CeNP, $n=3$ ) were euthanized for TEM analysis to evaluate white matter injury, and 12 mice $(\mathrm{ICH} 21 \mathrm{~d}+$ vehicle, $n=6$, ICH 21 days + PEG-CeNP, $n=6$ ) were undergone $\mathrm{MBP}$ and $\mathrm{dMBP}$ immunofluorescence staining. These aforementioned animals (TEM and MBP staining) were also applied to behavioral test on days 0 , $1,3,7,14$ and 21 days, $n=9$.

\section{Experiment 2}

In vivo and in vitro studies were used to investigate whether PEG-CeNP treatment modulated microglial polarization via inhibiting ROS-induced NF- $\mathrm{kB}$ p65 translocation. 30 mice were randomly divided into 5 groups: Sham group $n=6$, ICH 3 days $n=6, \mathrm{ICH} 3 \mathrm{~d}+$ PEG-CeNP $n=6$, ICH 7 days $n=6$, ICH 21 days $n=6$. Then, these animals were euthanized for PCR to detect the expression of M1/M2 microglia marker. In addition, 12 mice (ICH 3 days + vehicle $n=6$, ICH 3 days + PEG-
CeNP $n=6$ ) were undergone immunofluorescence staining (CD1632/Iba1, CD206/Iba1). Moreover, 24 mice (ICH 3 days + vehicle $n=12$, ICH 3 days + PEG-CeNP $n$ $=12$ ) were applied for flow cytometry and western blotting. The in vitro experiments were performed to further investigate the effects of PEG-CeNP on microglia.

\section{Experiment 3}

To investigate PEG-CeNP promoted OPC differentiation/maturation partly in a microglia-dependent manner, PLX3397 was used for microglia depletion in vivo. In total, 20 mice (Sham $n=5$, Sham $+\operatorname{PLX3397~} n=5$, ICH 7 days + vehicle $n=5$, ICH 7 days + PLX3397 $n=5$ ) were applied to flow cytometry and Iba1 immunostaining. Moreover, 24 mice (ICH 7 days + vehicle $n=6, \mathrm{ICH}$ 7 days + PEG-CeNP $n=6$, ICH 7 days + vehicle + PLX3397 $n=6$, ICH 7 days + PEG-CeNP + PLX3397 $n$ = 6) were undergone olig $2 / \mathrm{CC} 1$ immunostaining. In addition, 24 mice ( $\mathrm{ICH} 7$ days + vehicle $n=6, \mathrm{ICH} 7$ days + PEG-CeNP $n=6$, ICH 7 days + vehicle + PLX3397 $n=6$, ICH 7 days + PEG-CeNP + PLX3397 $n$ =6) were undergone EdU injection and the following EdU/CC1 immunostaining.

\section{Experiment 4}

In vivo and in vitro studies were used to investigate whether PEG-CeNP treatment protected against A1 astrocyte alteration after $\mathrm{ICH}$. A total of 30 mice were randomly divided into 4 groups: Sham group $n=6, \mathrm{ICH}$ 3 days $n=6$, ICH 7 days $n=6$, ICH 7 days + PEG-CeNP $n=6$, ICH $21 \mathrm{~d} n=6$. Then, these animals were euthanized for PCR to detect the expression of A1 astrocyte marker. Then, 12 mice (ICH 7 days + vehicle $n=6, \mathrm{ICH}$ 7 days + PEG-CeNP $n=6$ ) were undergone C3/GFAP immunofluorescence staining. In vitro, NF- $\mathrm{B}$ p65/ GFAP immunostaining and western blotting were performed to investigate whether PEG-CeNP treatment inhibited A1 astrocyte via inhibiting ROS-induced NFкB p65 translocation.

\section{Experiment 5}

To investigate whether A1 astrocytes inhibited microglial phagocytosis of myelin debris via an astrocytic C3microglial C3aR axis, Phagocytosis assay was performed by using fluorescent beads in vivo.

\section{Statistical analysis}

Results are shown as the mean \pm standard error of the mean (SEM). Student's $t$ test was used to compare 2 groups for continuous variables with normal distributions. The Mann-Whitney $U$ rank-sum test was used for continuous variables with non-normal distributions. For multiple groups, a one-way analysis of variance (ANOVA) with Tukey's post hoc analysis was used. A 
two-way ANOVA with Tukey's multiple comparisons test was used to assess the significance of behavioral tests in different groups at various time points. Data analysis was conducted by investigators blinded to experimental groups. Sample sizes for the animal studies were determined based on pilot studies or the current literature. Statistical Package for the Social Sciences (version 22.0) and Prism (version 8.0) were used for the statistical analysis. A $p$ value of $<0.05$ indicates statistical significance.

\section{Results}

\section{ICH-induced severe white and gray matter injury}

Nissl's staining showed that the injection of collagenase VII caused the striatum hemorrhage, which induced severe white and gray matter injury (Fig. 1a). The MBP immunofluorescence staining also showed significant structural damage in the myelin sheaths at 3 days post ICH (Fig. 1b).

CeNPs were modified with a PEG coating to have better biocompatibility and avoid interparticle agglomeration. As shown in Figure S2, PEG-CeNPs showed better colloidal stability than normal CeNPs after storage in pure water at $4{ }^{\circ} \mathrm{C}$. PEG-CeNP treatment significantly attenuated brain edema at 3 days after ICH (Fig. 1c, mean \pm SEM: $79.83 \pm 0.26 \%(\mathrm{ICH}+\mathrm{CeNP})$ versus 81.06 $\pm 0.38 \%$ (ICH + vehicle), $p<0.05)$. However, PEG-CeNP treatment failed to decrease the number of injured neurons (Fig. 1d, e, $p>0.05$ ).

\section{PEG-CeNP treatment reduced ROS accumulation}

To investigate the free radical scavenging activity of PEGCeNP in vivo, the superoxide-sensitive dye DHE was injected intraperitoneally at 3 days post ICH (Fig. 2a). Quantification of DHE-positive cells indicated that PEGCeNP treatment significantly reduced ROS accumulation (Fig. 2b, mean \pm SEM: $250.9 \pm 13.6 / \mathrm{mm}^{2}(\mathrm{ICH}+\mathrm{CeNP})$ versus $165.9 \pm 14.2 / \mathrm{mm}^{2}(\mathrm{ICH}+$ vehicle), $p<0.01)$. Likewise, the data of ROS assay in the brain tissues also supported the results mentioned above (Fig. $2 \mathrm{c}, \mathrm{ICH}+\mathrm{CeNP}$ versus ICH + vehicle, $p<0.01)$.

\section{PEG-CeNP treatment promoted remyelination and long- term neurological function}

To investigate whether PEG-CeNP treatment can promote remyelination, TEM analysis was performed to measure the thickness of the myelin sheaths at the perihematoma site in the striatum. Representative electron

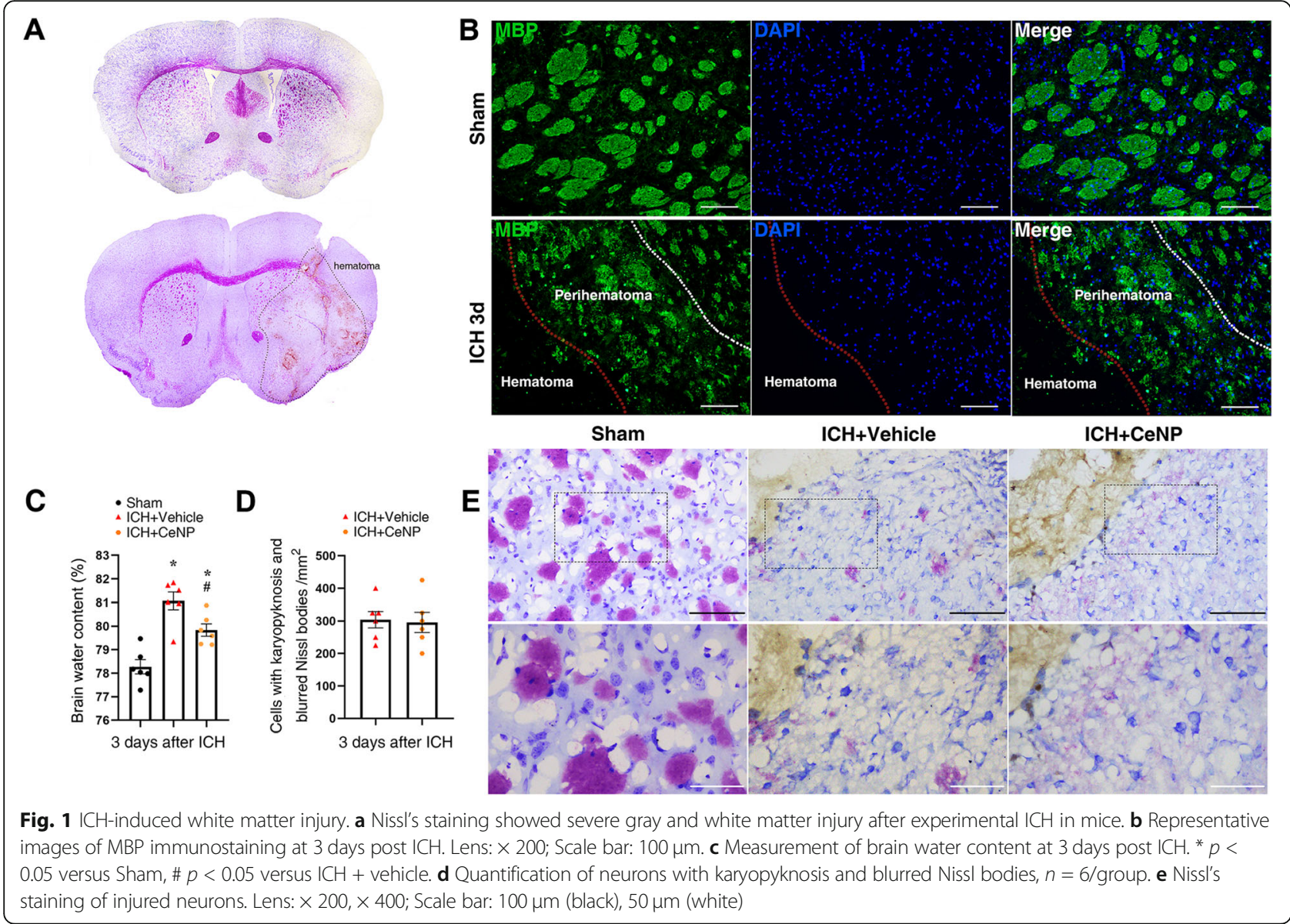



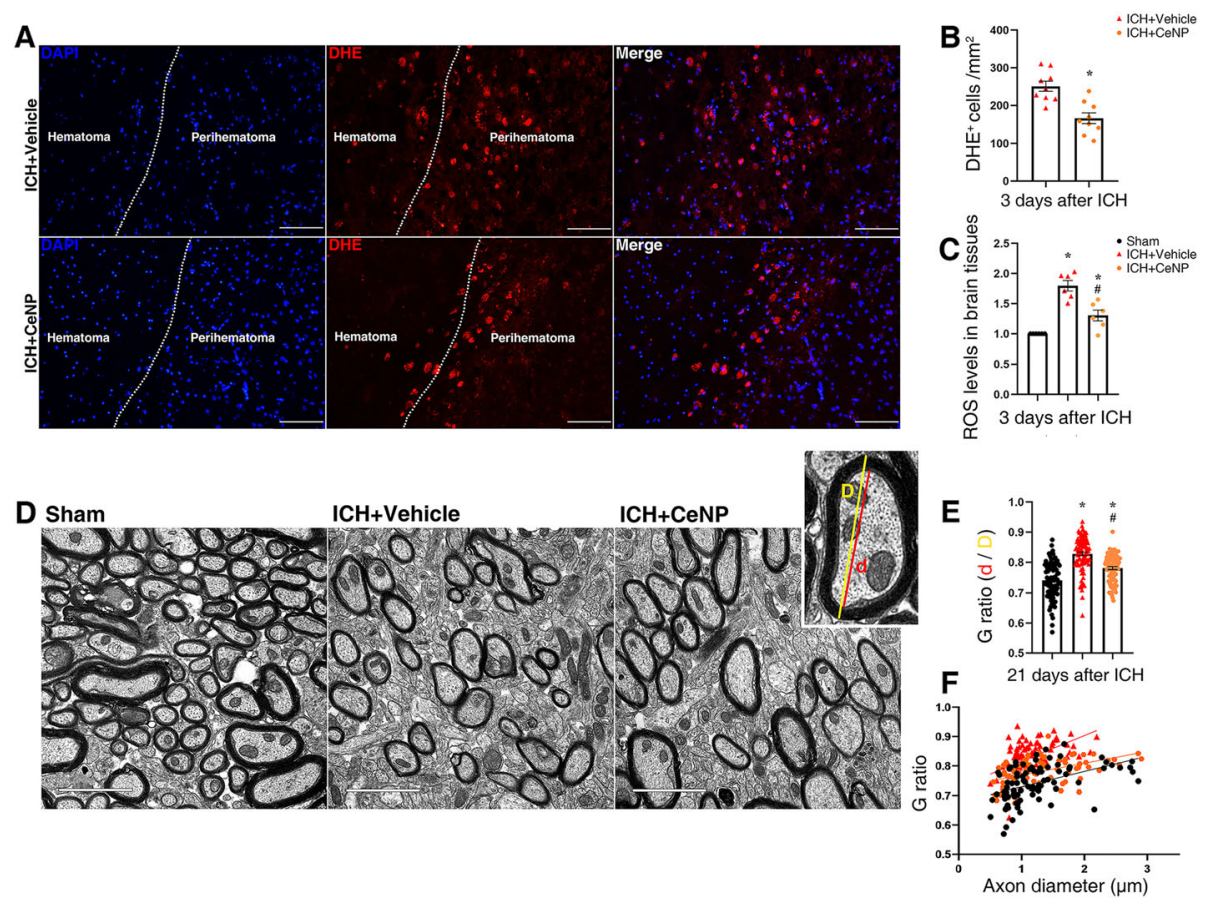

Fig. 2 PEG-CeNP treatment reduced ROS accumulation and improved anatomical integrity of myelinated fibers after ICH. a Representative images in peri-hematoma site stained with the superoxide-sensitive dye DHE at 3 days post ICH. Lens: $\times 200$; Scale bar: $100 \mu m$. $\mathbf{b}$ Quantification of DHE+DAPI+ cells, $n=3$ /group, ${ }^{*} p<0.01$ versus $\mathrm{ICH}+$ vehicle. c ROS levels in brain tissues at 3 days post $\mathrm{ICH}, n=6 /$ group, ${ }^{*} p<0.01$ versus Sham, \# $p<0.01$ versus ICH + vehicle. $\mathbf{d}$ Representative electron micrographs showed myelin sheaths in striatum at 21 days post ICH. Lens: $\times 15,000 ;$ Scale bar: $2 \mu m$. e Histograms show quantifications of the G-ratio at 21 days post ICH, $n=3 /$ group, ${ }^{*} p<0.01$ versus Sham, \# $p<0.01$ versus ICH + vehicle. f Scatter plots of G-ratio against axon diameter

micrographs showed the myelin sheaths in the perihematoma region at 21 days post ICH (Fig. 2d). The results showed that the myelin sheaths in ICH mice displayed a higher G-ratio than those in the sham group (Fig. 2e, f, $p<0.01$ versus sham, average G-ratio: sham $0.74 \pm 0.0063, \mathrm{ICH}+$ vehicle $0.82 \pm 0.006, \mathrm{ICH}+\mathrm{CeNP}$ $0.78 \pm 0.005)$, indicating a reduction in the myelin thickness. By contrast, mice in the $\mathrm{ICH}+\mathrm{CeNP}$ group showed a lower G-ratio compared to the ICH mice (Fig. 2e, f, $p$ $<0.01$ versus $\mathrm{ICH}+$ vehicle). Representative imaging of the MBP immunostaining displayed the area of the white matter lesion at 21 days post ICH (Fig. 3a). PEG-CeNP treatment significantly decreased the white matter lesion compared to $\mathrm{ICH}+$ vehicle (Fig. $3 \mathrm{~b}, p<0.01$ versus $\mathrm{ICH}$ + vehicle). Namely, PEG-CeNP treatment promoted remyelination and improved anatomical integrity of the myelinated fibers.

Adhesive removal test was applied 1, 3, 7, 14, and 21 days after ICH. PEG-CeNP-treated mice and vehicletreated mice displayed a significant reduction in contact and removal time compared to the sham group (Fig. 3c, $\mathrm{d}, p<0.01$ versus sham). This indicated that ICH caused significant sensorimotor deficit. PEG-CeNP treatment significantly alleviated such sensorimotor deficit at 7 and 14 days post $\mathrm{ICH}$ (Fig. 3c, d, $p<0.05$ versus $\mathrm{ICH}+$ vehicle). The accelerated rotarod test showed that the motor coordination and limb strength of mice were significantly decreased after ICH induction in both PEGCeNP-treated mice and vehicle-treated mice (Fig. 3e, $p<$ 0.01 versus sham). Long-term motor coordination and limb strength in the rotarod test at 14 and 21 days post $\mathrm{ICH}$ were significantly improved in PEG-CeNP-treated mice (Fig. 3e, $p<0.01$ versus $\mathrm{ICH}+$ vehicle).

\section{PEG-CeNP treatment-induced microglia skewed polarization}

To investigate the polarization dynamics of microglia after ICH, RT-PCR was used to test the mRNA expression of M1 and M2 microglia markers in the brain tissue 3, 7, and 21 days after ICH. As shown in Fig. $4 a-c$, the mRNA expression of these classic M2 markers (CD206, YM1/2, TGF- $\beta)$ peaked at 3 or 7 days after ICH $(p<$ 0.01 ). The difference became less obvious at 21 days post ICH (Fig. $4 \mathrm{a}-\mathrm{c}, p>0.05$ versus sham). The mRNA expression of these classic M1 markers, CD16 and iNOS, peaked at 3 or 7 days after ICH, except for IFN- $\gamma$ (Fig. $4 \mathrm{~d}-\mathrm{f}, p<0.01)$. However, the mRNA expression of IFN$\gamma$ increased after ICH induction ( $p<0.01$ versus sham). The difference between these three groups (ICH 3, 7, and 21 days) was not statistically significant $(p>0.05)$. 


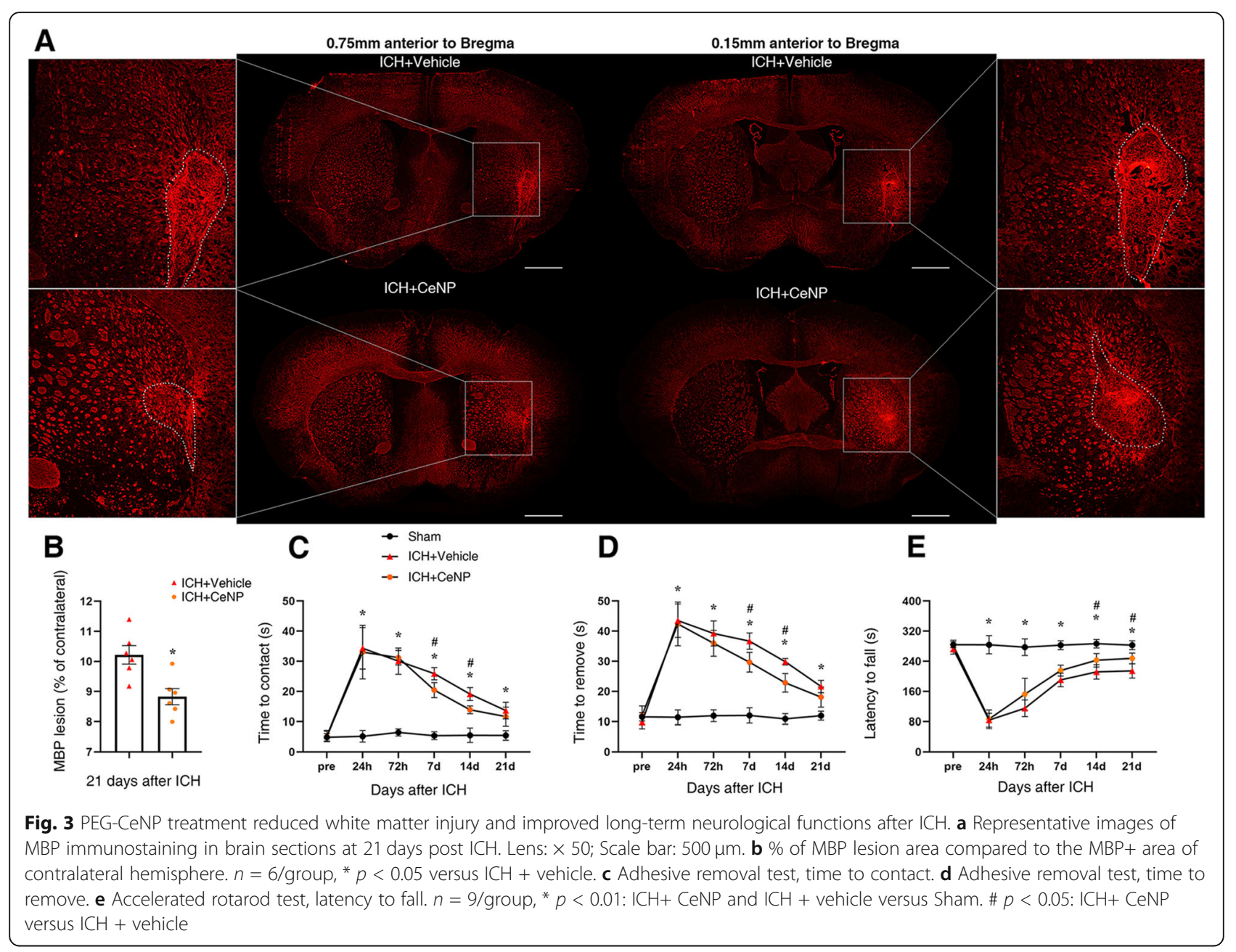

In light of the results mentioned above, day 3 was chosen as the specific time point to explore the effects of PEG-CeNP in ICH mice. PEG-CeNP treatment significantly inhibited CD16 mRNA expression compared to $\mathrm{ICH}+$ vehicle (Fig. $4 \mathrm{~h}, p<0.01$ versus $\mathrm{ICH}+$ vehicle). In addition, CD206 mRNA expression was slightly increased in PEG-CeNP-treated mice (Fig. 4i, $p<0.05$ versus $\mathrm{ICH}+$ vehicle).

Since the mRNA expression in brain tissue could not specifically reflect the changes in microglia, immunofluorescence staining was conducted to identify the expression of M1 and M2 markers in microglia after ICH (Fig. 4g). The immunofluorescence staining of CD206 and $\mathrm{CD} 16 / 32$ at 3,7 , and 21 days post ICH showed good consistency with mRNA expression time curves (Fig. $4 \mathrm{~g}$ ICH 3 days, Figure S3 ICH 7 days, Figure S4 ICH 21d). The number of $\mathrm{CD} 16 / 32^{+} \mathrm{Iba}^{+}$cells were significantly decreased in PEG-CeNP-treated mice (Fig. 4j, mean \pm SEM: $485 \pm 19.57(\mathrm{ICH}+$ vehicle $)$ versus $344 \pm 17.16$ $(\mathrm{ICH}+\mathrm{CeNP}), p<0.01)$. $\mathrm{CD}^{206^{+}} \mathrm{Iba1}^{+}$cells were moderately increased in ICH mice with PEG-CeNP treatment (Fig. $4 \mathrm{k}$, mean \pm SEM: $70 \pm 5.33(\mathrm{ICH}+$ vehicle) versus $90 \pm 4.89(\mathrm{ICH}+\mathrm{CeNP}), p<0.05)$.

\section{PEG-CeNP treatment modulated microglial polarization via inhibiting ROS-induced NF-KB p65 translocation}

Flow cytometric analysis was performed to quantify further the proportion of M1 and M2 microglia in the brain tissue. The representative gating strategy for microglia $\left(\mathrm{CD} 45^{\text {low }+} \mathrm{CD}_{11 \mathrm{~b}^{+}}\right)$, macrophages $\left(\mathrm{CD} 45^{\text {high }+} \mathrm{CD} 11 \mathrm{~b}^{+}\right)$, M1 microglia $\left(\mathrm{CD} 16 / 32^{+} \mathrm{CD} 206^{-}, \mathrm{CD} 45^{\text {low }}{ }^{\mathrm{C}} \mathrm{CD} 11 \mathrm{~b}^{+}\right)$, and M2 microglia $\left(\mathrm{CD} 16 / 32^{-} \mathrm{CD} 206^{+}, \mathrm{CD} 45^{\text {low+ }}{ }^{+} \mathrm{CD} 11 \mathrm{~b}^{+}\right)$ is shown in Fig. 5a. The quantity of $\mathrm{CD} 16 / 32^{+} \mathrm{CD} 206^{-}$ microglia were decreased after PEG-CeNP treatment compared to $\mathrm{ICH}+$ vehicle (Fig. 5b, $p<0.05$ ). PEGCeNP treatment also increased the number of CD16/ $32^{-} \mathrm{CD}_{206}{ }^{+}$microglia (Fig. $5 \mathrm{c}, p<0.05$ versus $\mathrm{ICH}+$ vehicle). The mean fluorescence intensity of CD206 was also in accordance with the results mentioned previously (Fig. 5d).

Microglial phenotypes are tightly controlled by ROS levels. As a traditional transcription factor, NF- $\kappa B$ plays 

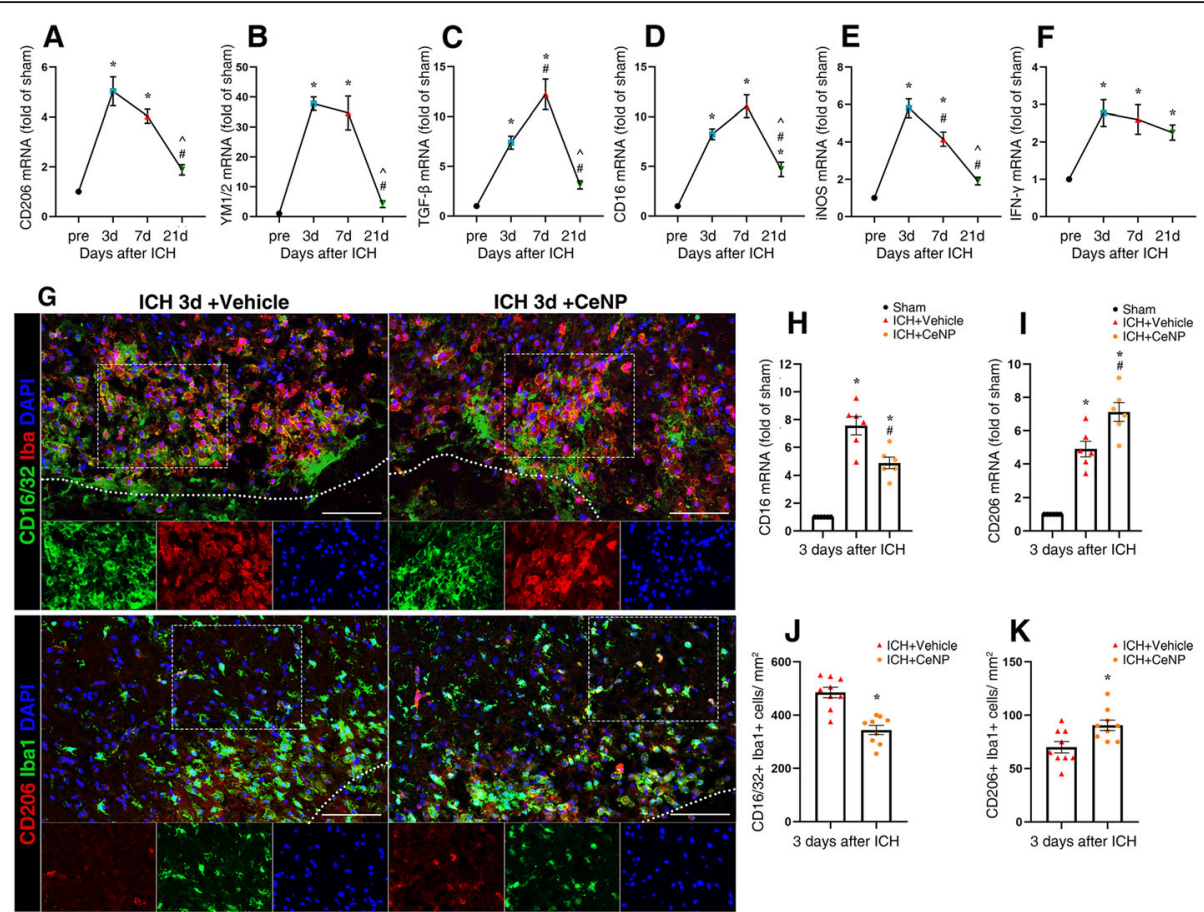

Fig. 4 PEG-CeNP treatment-induced microglia skewed polarization after ICH. Representative RT-qPCR analysis of M2 (a-c) and M1 (d-f) microglia markers expression. $n=6 /$ group, ${ }^{*} p<0.01$ versus Sham. $\# p<0.01$ versus ICH 3d. $\wedge p<0.01$ versus ICH 7d. $\mathbf{g}$ Representative images of CD1632/ Iba1 and CD206/lba1 double immunostaining at 3 days post ICH. Lens: $\times$ 200; Scale bar: 100 mm. h CD16 mRNA expression in PEG-CeNP-treated mice at 3 days post $\mathrm{ICH} . n=6 /$ group, ${ }^{*} p<0.01$ versus Sham, $\# p<0.01$ versus $\mathrm{ICH}+$ vehicle. $\mathbf{i}$ CD206 mRNA expression in PEG-CeNP-treated mice at 3 days post ICH. $n=6 /$ group, ${ }^{*} p<0.01$ versus Sham, \# $p<0.05$ versus $\mathrm{ICH}+$ vehicle. $\mathbf{j}$ Quantification of $\mathrm{CD} 16 / 32^{+} \mid \mathrm{Iba} 1^{+}$cells, $n=6 /$ group, ${ }^{*} p<0.01$ versus $\mathrm{ICH}+$ vehicle. $\mathbf{k}$ Quantification of $\mathrm{CD}^{206}{ }^{+} \mid \mathrm{ba} 1^{+}{ }^{+}$cells, $n=6 /$ group, ${ }^{*} p<0.05$ versus ICH + vehicle

a crucial role in microglia activation. ROS can induce NF- $\mathrm{KB}$ p65 activation and translocation, and then regulate microglia polarization $[11,31]$. Hence, we detected the protein expression of phosphorylated NF- $\mathrm{kB}$ p65 (Pp65) and p65 in mice brain tissue after ICH induction. The western blot results indicated that $\mathrm{ICH}$ significantly increased P-p65 expression (Fig. 5e, f, $p<0.05$ versus sham), and PEG-CeNP treatment decreased P-p65 expression (Fig. 5e, f, $p<0.05$ versus $\mathrm{ICH}+$ vehicle).

In cultured microglia, $\mathrm{H}_{2} \mathrm{O}_{2}$ was used to induce microglia activation and ROS accumulation. As shown in Fig. $5 \mathrm{~g}$, PEG-CeNP displayed remarkable free radical scavenging activity in vitro. ROS levels in microglia treated with $\mathrm{H}_{2} \mathrm{O}_{2}$ were significantly reduced after PEG-CeNP treatment ( $p<0.05$ versus $\mathrm{H}_{2} \mathrm{O}_{2}+$ vehicle). Representative images of NF-KB p65 and Iba1 double immunostaining in cultured microglia indicated that $\mathrm{H}_{2} \mathrm{O}_{2}$ treatment resulted in obvious translocation of NF- $\mathrm{kB}$ p 65 from the cytoplasm to the nucleus (Fig. 5i). Also, the western blot analysis revealed that ROS accumulation induced the downregulation of IкB- $\alpha$ (inhibitor of NF- $\mathrm{kB}$ ) and cytoplasmic p65 in microglia (Fig. $5 \mathrm{~h}, \mathrm{k}, p<0.05$ versus control). In contrast, nuclear NF-kB p65 was significantly increased in microglia after $\mathrm{H}_{2} \mathrm{O}_{2}$ stimulation (Fig. $5 \mathrm{j}, p$ $<0.05$ versus control). PEG-CeNP treatment inhibited
I $\kappa \mathrm{B}-\alpha$ degradation and the translocation of NF- $\kappa \mathrm{B}$ p 65 from the cytoplasm to the nucleus (Fig. 5h, j, k, $p<0.05$ versus $\mathrm{H}_{2} \mathrm{O}_{2}+$ vehicle).

\section{PEG-CeNP promoted OPCs differentiation, partly in a microglia-dependent manner}

Previous studies demonstrated that M2 microglia could promote oligodendrocyte differentiation, which was essential for remyelination in the CNS [12, 32]. PLX3397 (an inhibitor of colony-stimulating factor 1 receptor) was administrated to deplete microglia to investigate whether PEG-CeNP promotes remyelination in a microglia-dependent manner (Fig. 6a). The flow cytometry (Fig. 6b) and immunofluorescence staining (Fig. 6c) results showed that PLX3397 achieved good depletion efficacy in both the sham (Fig. 6d, mean \pm SEM: $136 \pm$ 8.23 versus $37 \pm 2.59, p<0.01$ versus sham) and $\mathrm{ICH}$ mice (Fig. 6e, mean \pm SEM: $884 \pm 34.08$ versus $324 \pm$ $31.00, p<0.01$ versus $\mathrm{ICH})$, respectively. The electron micrograph showed the essential role of mature oligodendrocyte in myelination (Fig. 6f).

The CC-1 and Olig2 double immunostaining images showed the quantity of mature oligodendrocytes $\left(\mathrm{CC}-1^{+}\right.$ Olig2 ${ }^{+}$) in the peri-hematoma region at 7 days post $\mathrm{ICH}$ (Fig. 6g). Mature oligodendrocytes were significantly 

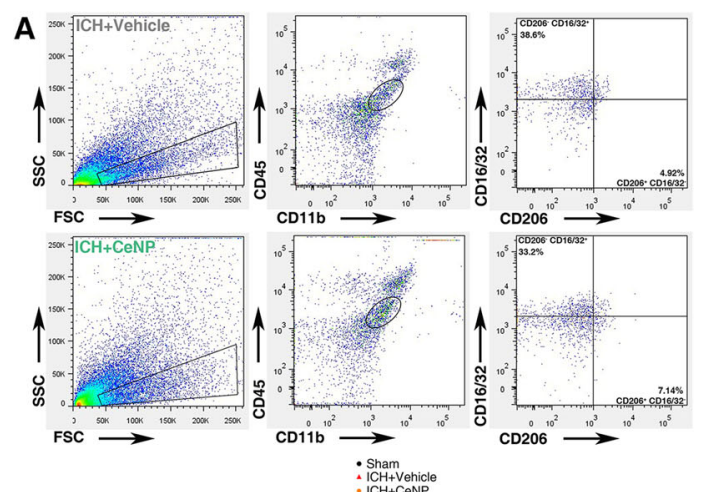

C
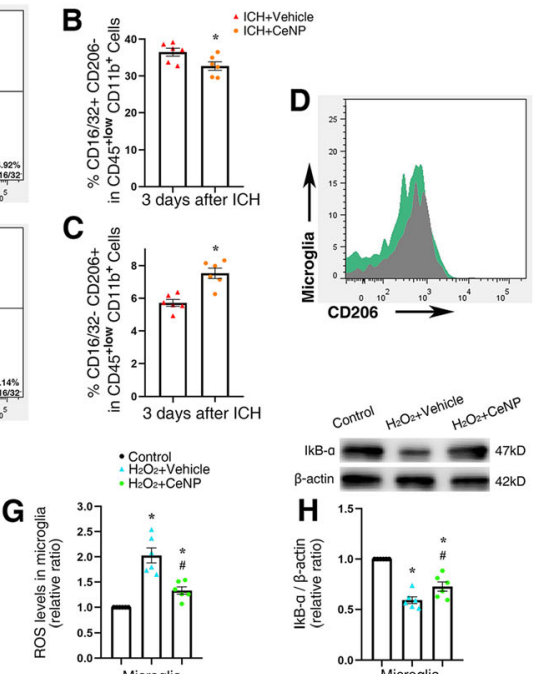

$\cdot \mathrm{H}_{2} \mathrm{O}_{2}+\mathrm{CeNP}$
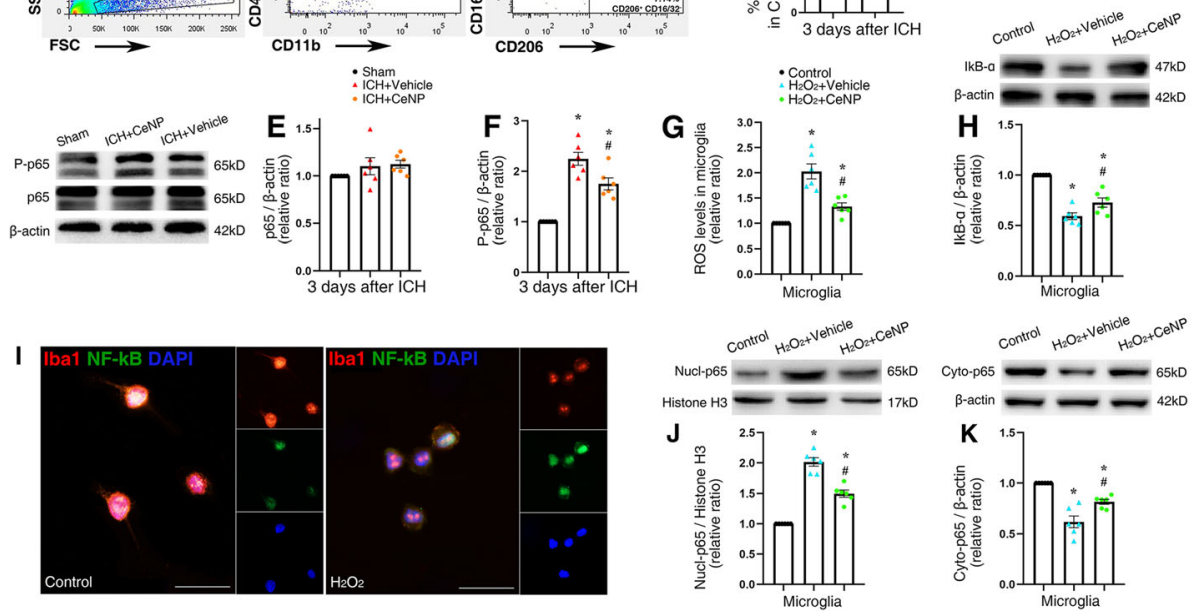

Fig. 5 PEG-CeNP treatment modulated microglial polarization via inhibiting ROS-induced NF-kB p65 translocation. a Flow cytometric analysis of microglia isolated from the ipsilateral hemisphere at 3 days post ICH. Representative gating strategy for microglia $\left(C D 45^{\text {low+ }} \mathrm{CD} 11 \mathrm{~b}^{+}\right)$, macrophages $\left(\mathrm{CD} 45^{\mathrm{high}+} \mathrm{CD} 11 \mathrm{~b}^{+}\right)$,

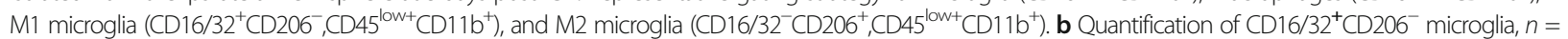
6/group, ${ }^{*} p<0.01$ versus ICH + vehicle. c Quantification of CD16/32- CD206 ${ }^{+}$microglia, $n=6 /$ group, ${ }^{*} p<0.05$ versus ICH + vehicle. $\mathbf{d}$ Mean fluorescence intensity of CD206 in ICH + vehicle (gray) and ICH+ CeNP (green) group. e, $\mathbf{f}$ Representative Western blot bands showed the expression of NF-kB p65 and phosphorylated NF-KB p65 (P-p65) in brain tissues at 3 days after ICH, $n=6 /$ group, ${ }^{*} p<0.05$ versus Sham, $\# p<0.05$ versus ICH + vehicle. $\mathbf{g}$ ROS levels in cultured microglia, $n=6 /$ group, ${ }^{*} p<0.05$ versus Control, $\# p<0.05$ versus $\mathrm{H}_{2} \mathrm{O}_{2}+$ vehicle. $\mathbf{h}$ Representative Western blot bands showed the expression of IkBa in cultured microglia, $n=6 /$ group, ${ }^{*} p<0.05$ versus Control, $\# p<0.05$ versus $\mathrm{H}_{2} \mathrm{O}_{2}+$ vehicle. i Representative images of NF-kB p65 and Iba1 double immunostaining in cultured microglia. Lens: $\times 400$; Scale bar: $25 \mu \mathrm{m}$. j, k Representative Western blot bands showed the expression of nuclear and cytoplasm $\mathrm{NF}-\mathrm{KB}$ p65 in cultured microglia, $n=6 /$ group, ${ }^{*} p<0.05$ versus Control, $\# p<0.05$ versus $\mathrm{H}_{2} \mathrm{O}_{2}+$ vehicle

increased in PEG-CeNP-treated mice (Fig. 6h, mean \pm SEM: $256 \pm 12.16(\mathrm{ICH}+\mathrm{CeNP})$ versus $209 \pm 8.99(\mathrm{ICH}$ + vehicle), $p<0.01)$. Meanwhile, the number of CC- $1^{+}$ Olig2 ${ }^{+}$cells was significantly decreased after PLX3397 administration (Fig. 6h, mean \pm SEM: $86 \pm 9.60(\mathrm{ICH}+$ vehicle + PLX3397) versus $209 \pm 8.99$ (ICH + vehicle), $p$ $<0.01$ ). PLX3397 administration also decreases the number of oligodendrocyte-lineage cells Olig2 $^{+}$cells, Figure S5A). Interestingly, we found that the number of mature oligodendrocytes was moderately enhanced in $\mathrm{ICH}+\mathrm{CeNP}+$ PLX3397-treated mice when compared with the ICH + vehicle + PLX3397 group (Fig. 6h, mean \pm SEM: $142 \pm 4.89(\mathrm{ICH}+\mathrm{CeNP}+\mathrm{PLX} 3397)$ versus 86 $\pm 9.60(\mathrm{ICH}+$ vehicle + PLX3397), $p<0.05)$.

In order to label proliferating cells, mice were intraperitoneally injected with a thymidine analog (EdU). The EdU and CC-1 double immunostaining images showed that the newly differentiated mature oligodendrocytes were significantly increased in ICH + CeNP-treated mice at 7 days after ICH (Fig. 6i, j, mean \pm SEM: $50.83 \pm 5.72$ $(\mathrm{ICH}+$ vehicle) versus $70 \pm 6.91(\mathrm{ICH}+\mathrm{CeNP}), p<$ 0.05). PLX3397 administration decreased the number of proliferating cells $\left(\mathrm{EdU}^{+}\right.$cells, Figure S5B). Meanwhile, PEG-CeNP treatment also moderately promoted the expression of $\mathrm{EdU}^{+}$and $\mathrm{CC}^{-}{ }^{+}$cells in $\mathrm{ICH}+\mathrm{CeNP}$ +PLX3397-treated mice compared to $\mathrm{ICH}+$ vehicle + PLX3397 group (Fig. 6i, j, mean \pm SEM: $55.83 \pm 5.31$ $(\mathrm{ICH}+\mathrm{CeNP}+\mathrm{PLX} 3397)$ versus $28.33 \pm 6.91(\mathrm{ICH}+$ vehicle + PLX3397), $p<0.05)$.

In light of the results mentioned above, we assumed that PEG-CeNP promoted OPCs differentiation, partly in a microglia-dependent manner, and astrocytes might also participate in the pathophysiological process.

\section{ICH-induced A1 astrocyte alteration}

As we know, A1 astrocytes lose most normal astrocytic functions and exert a neurotoxic function that rapidly devastate mature, differentiated oligodendrocytes [14]. 

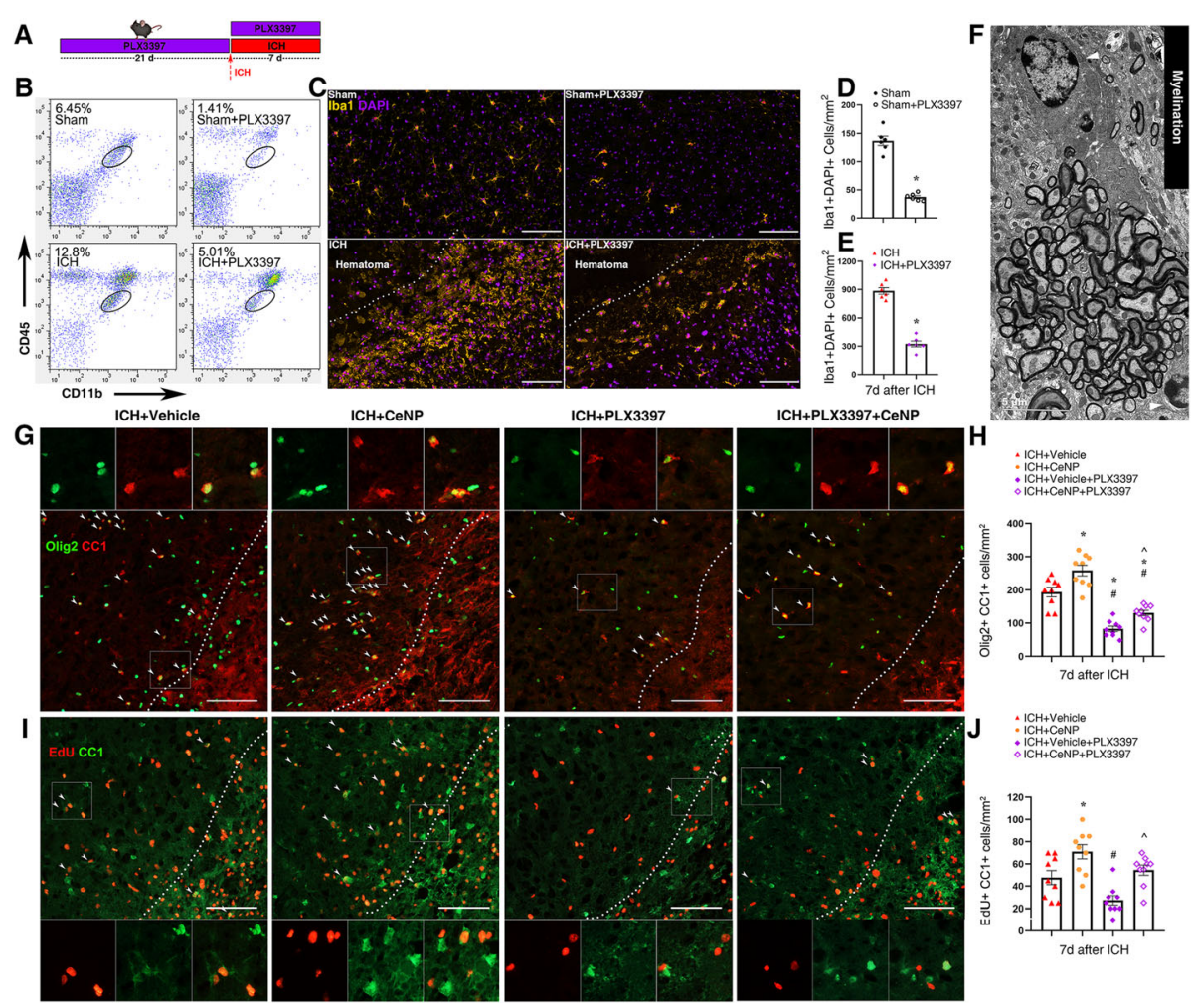

Fig. 6 PEG-CeNP promoted OPCs differentiation/maturation partly in a microglia-dependent manner. a PLX3397 administration. b Representative flow cytometric dot-plot showed the percentage of microglia after PLX3397 administration in different groups. c Representative fluorescent images showed the expression of Iba $1^{+}$Cells after PLX3397 administration in striatum. Lens: $\times 200$; Scale bar: $100 \mu \mathrm{m}$. d Quantification of Iba1 ${ }^{+}$ Cells in Sham and Sham+PLX3397 group, $n=3$ /group, ${ }^{*} p<0.01$ versus Sham. e Quantification of Iba ${ }^{+}$Cells in ICH and ICH + PLX3397 group, $n$ $=3 /$ group, ${ }^{*} p<0.01$ versus ICH. $\mathbf{f}$ Representative TEM image of myelination showed the ultra-structures of oligodendrocyte (white arrows) and myelin sheath. Lens: $\times$ 7500; Scale bar: $5 \mu \mathrm{m}$. g Representative images of olig2 and CC1 double immunostaining at 7 days post ICH. Lens: $\times 200$; Scale bar: $100 \mu \mathrm{m}$. h Quantification of $\mathrm{CC}^{+}{ }^{+}$olig2 ${ }^{+}$cells, $n=6 /$ group, $^{*} p<0.01$ versus $\mathrm{ICH}+$ vehicle, $\# p<0.01$ versus ICH $+\mathrm{CeNP}, \wedge p<0.05$ versus ICH + vehicle+PLX3397. i Representative images of EdU and CC1 double immunostaining at 7 days post ICH. Lens: $\times 200$; Scale bar: $100 \mu \mathrm{m}$. j Quantification of EdU ${ }^{+} \mathrm{CC}^{+}$cells, $n=6 /$ group, $^{*} p<0.05$ versus $\mathrm{ICH}+$ vehicle, $\# p<0.05$ versus ICH $+\mathrm{CeNP}, \wedge p<0.05$ versus ICH + vehicle+PLX3397

To investigate the profile of astrocyte alteration during the ICH process, RT-PCR was used to examine the mRNA expression of A1 astrocyte markers in the brain tissue at 3, 7, and 21 days after $\mathrm{ICH}$. As the most characteristic marker in A1 astrocytes, the mRNA expression of complement component 3 (C3) was significantly elevated (approximately 50-200 folds higher than sham) and peaked at 7 days after ICH induction (Fig. 7a, $p<$ 0.01). In addition, $\mathrm{ICH}$ also induced the upregulation of Amigo2 and Serping1 mRNA expression (Fig. 7b, c, $p<$ 0.01). Representative images of C3 and GFAP double staining at 3,7 , and 21 days post $\mathrm{ICH}$ were also in accordance with the tendency of C3 mRNA expression (Figure S6).

PEG-CeNP treatment hindered $\mathrm{A} 1$ astrocyte alteration via inhibiting ROS-induced NF-KB p65 translocation

As shown in Fig. 7d, PEG-CeNP treatment resulted in the downregulation of $\mathrm{C} 3 \mathrm{mRNA}$ expression after
$\mathrm{ICH}(p<0.01$ versus $\mathrm{ICH}+$ vehicle $)$. Likewise, the $\mathrm{C} 3$ and GFAP double staining image also indicated that PEG-CeNP treatment significantly decreased A1 astrocyte expression at 7 days post ICH (Fig. 7f). Previous studies demonstrated that ROS might induce astrocyte activation [20], and A1 reactive astrocyte might be induced by NF-kB signaling [33, 34]. Hence, the cultured astrocytes were treated with $\mathrm{H}_{2} \mathrm{O}_{2}$ to investigate whether ROS could induce $\mathrm{A} 1$ reactive astrocyte directly. We found that $\mathrm{H}_{2} \mathrm{O}_{2}$ treatment strongly induced A1 astrocyte (Fig. 5e) compared to the control group (Figure S7). Simultaneously, the C3 mRNA expression also strongly increased in $\mathrm{H}_{2} \mathrm{O}_{2}$ treated astrocytes (Fig. $7 \mathrm{~g}, p<0.05$ versus control), and PEG-CeNP treatment reversed the change (Fig. $7 \mathrm{~g}, p<0.05$ versus $\mathrm{H}_{2} \mathrm{O}_{2}+$ vehicle). The western blot analysis showed that nuclear NF-kB p65 was significantly increased in astrocytes after $\mathrm{H}_{2} \mathrm{O}_{2}$ stimulation (Fig. $7 \mathrm{~h}, \quad p<0.05$ versus control). PEG-CeNP 

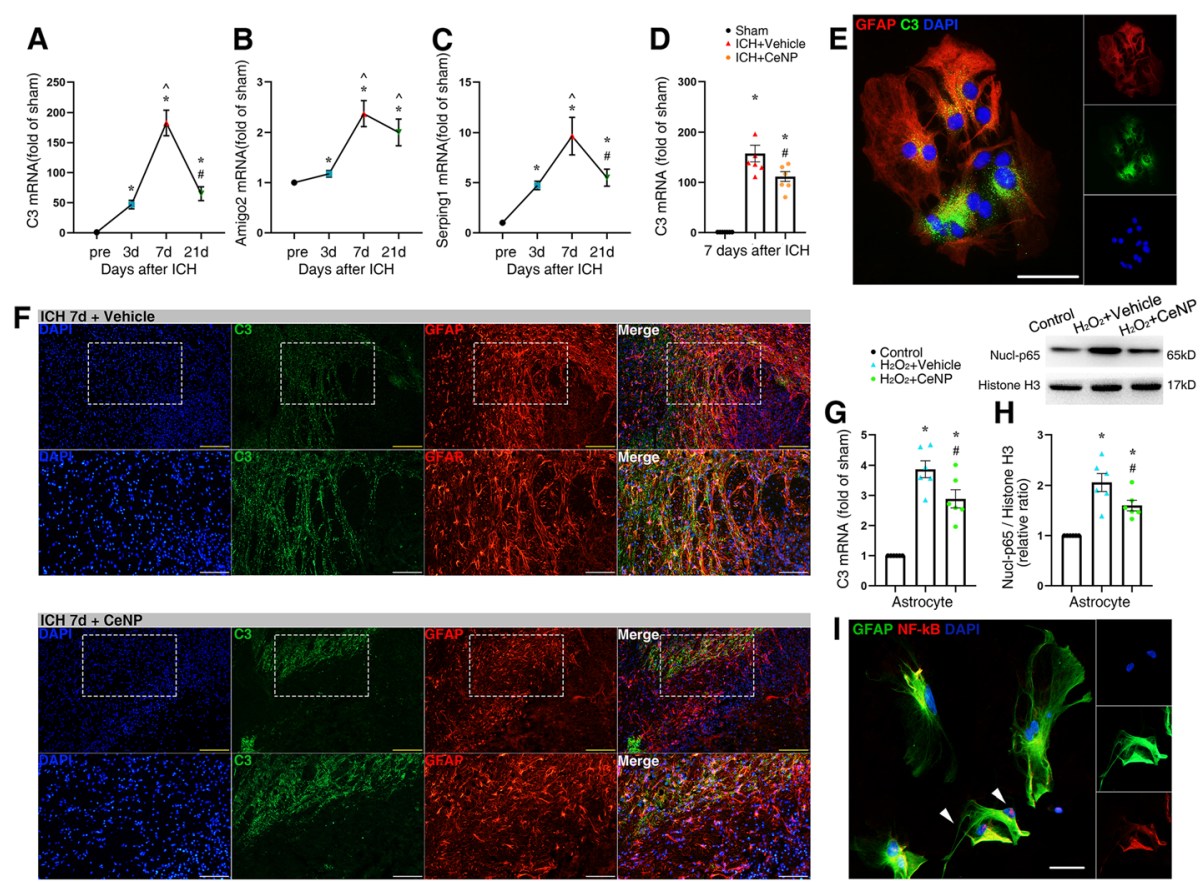

Fig. 7 PEG-CeNP treatment protected against A1 astrocyte alteration after ICH. Representative RT-qPCR analysis of A1 astrocyte markers expression. a C3 mRNA expression. b Amigo2 mRNA expression. c Serping1 mRNA expression. $n=6 /$ group, ${ }^{*} p<0.01$ versus Sham. $\wedge p<0.01$ versus ICH $3 \mathrm{~d}$. \# $p<0.01$ versus ICH $7 \mathrm{~d}$. d C3 mRNA expression in PEG-CeNP-treated mice at 7 days post ICH. $n=6 /$ group, ${ }^{*} p<0.01$ versus Sham, $\# p<0.01$ versus ICH + vehicle. e Representative images of GFAP and C3 double immunostaining in cultured astrocytes (treated with $\mathrm{H}_{2} \mathrm{O}_{2}$ ). Lens: $\times 400$; Scale bar: $50 \mu$ m. f Representative images of GFAP and C3 double immunostaining in brain sections at 7 days post ICH. Lens: $\times 100, \times 200$; Scale bar: $200 \mu$ m (yellow), $100 \mu m$ (white). g C3 mRNA expression in cultured astrocytes. $n=6$ /group, ${ }^{*} p<0.05$ versus Control, $\# p<0.05$ versus $\mathrm{H}_{2} \mathrm{O}_{2}+$ vehicle. $\mathbf{h}$ Representative Western blot band showed the expression of nuclear NF-kB p65 in cultured astrocytes, $n=6 /$ group, ${ }^{*} p<0.05$ versus Control, \# $p<0.05$ versus $\mathrm{H}_{2} \mathrm{O}_{2}+$ vehicle. i Representative images of NF-kB p65 and GFAP double immunostaining in cultured astrocytes (treated with $\mathrm{H}_{2} \mathrm{O}_{2}$ ). Lens: $\times 400$; Scale bar: $25 \mu \mathrm{m}$

treatment inhibited the translocation of NF- $\mathrm{kB}$ p 65 from the cytoplasm to the nucleus (Fig. $7 \mathrm{~h}, p<0.05$ versus $\mathrm{H}_{2} \mathrm{O}_{2}+$ vehicle). Representative images of NF$\mathrm{\kappa B}$ p65 and GFAP double immunostaining in cultured astrocytes also indicated that $\mathrm{H}_{2} \mathrm{O}_{2}$ treatment resulted in the translocation of NF-kB p65 from the cytoplasm to the nucleus (Fig. 7i) compared to the control group (Figure S8).

\section{Astrocytes communicated with microglia via an astrocytic C3-microglial C3aR axis after ICH}

Astrocyte and microglia have a unique bond and always coordinate their functions when the brain is perturbed [13]. Previous studies demonstrated that plenty of activated microglia were embedded in a GFAP-rich glial scar, and rapidly increased their phagocytic capacity to remove debris after CNS injury [13, 35]. The same phenomenon was also observed in the experimental $\mathrm{ICH}$ model (Fig. 8a). An astrocytic scar was formed to enclose the hematoma lesion and restricted the development of neuroinflammation. The embedded microglia exerted their phagocytic capacity to remove myelin debris, which can be seen in the electron micrograph (Fig. $8 \mathrm{~b})$. dMBP immunostaining was used to investigate whether PEG-CeNP treatment increases the microglial engulfment of myelin debris in vivo. As shown in Figure S9, PEG-CeNP treatment effectively reduced the percentage of $\mathrm{dMBP}$ area compared to $\mathrm{ICH}+$ vehicle at 3 days after $\mathrm{ICH}(p<0.05$, mean \pm SEM: $6.07 \pm 0.56 \%$ $(\mathrm{ICH}+$ vehicle $)$ versus $4.30 \pm 0.38 \%(\mathrm{ICH}+\mathrm{CeNP}))$. Under the physiological condition, C3 could mediate the microglial engulfment of the redundant spines to ensure synaptic refinement via the $\mathrm{C} 3 / \mathrm{C} 3 \mathrm{aR}$ signaling pathway $[29,36]$. As shown in Fig. 8c, d, the data from a singlecell transcriptome database indicated that the $\mathrm{C} 3 \mathrm{a}$ receptor (C3aR) is strongly expressed in microglia (Tabula Muris). The immunostaining image of brain tissue cryosection also showed that $\mathrm{C} 3 \mathrm{aR}$ was abundantly expressed in microglia (Fig. 8e). Since A1 astrocyte highly expressed C3, fluorescent beads were used to investigate whether astrocytes mediated microglial phagocytosis via an astrocytic C3-microglial C3aR axis (Fig. 8g). Microglia showed good phagocytosis in the control group, C3 and A1 astrocyte CM treatment strongly diminished the bead uptake (Fig. 8f, g, $p<0.01$ versus control). However, C3aR antagonist (C3aRA, SB290157) administration could reverse the negative effects of C3 and A1 astrocyte $\mathrm{CM}$ in microglial phagocytosis (Fig. 8f, g, $p<$ 


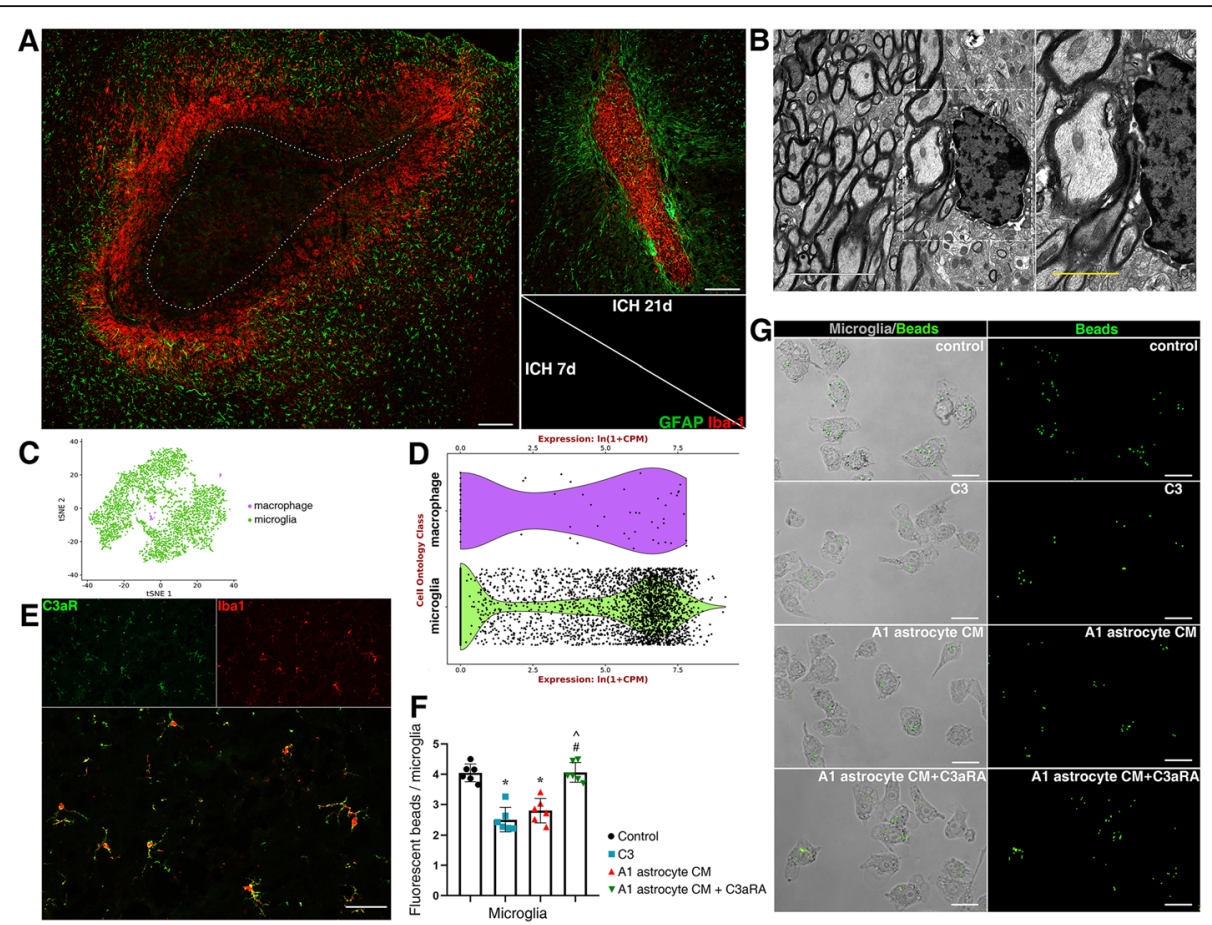

Fig. 8 A1 astrocytes inhibited microglial phagocytosis of myelin debris via an astrocytic C3-microglial C3aR axis. a Representative images of GFAP and Iba1 double immunostaining in brain sections. Lens: $\times 100$; Scale bar: $200 \mu \mathrm{m}$. b Representative TEM image showed microglia phagocytosis of myelin debris. Lens: $\times$ 7500, $\times$ 15,000; Scale bar: $5 \mu \mathrm{m}$ (white), $2 \mu \mathrm{m}$ (yellow). c tSNE plots of brain myeloid cells (Tabula Muris). $\mathbf{d}$ Expression of C3aR1 genes in microglia and macrophage (Tabula Muris). e Representative images of C3aR and Iba1 double immunostaining in brain sections. Lens: $\times 200 ;$ Scale bar: $50 \mu \mathrm{m}$. f Quantification of fluorescent beads in cultured microglia, ${ }^{*} p<0.01$ versus Control, \# $p<0.01$ versus C3 treatment, $\wedge p<0.01$ versus A1 astrocyte CM treatment. $\mathbf{g}$ Representative images of fluorescent beads uptake in cultured microglia. Lens: $\times$ 400; Scale bar: $25 \mu \mathrm{m}$

0.01 versus $\mathrm{C} 3$ and $\mathrm{A} 1$ astrocyte $\mathrm{CM})$. These results indicate that an astrocytic C3-microglial C3aR axis plays a crucial role in astrocyte-microglia crosstalk.

\section{Discussion}

Since free radicals play a prominent role in the pathological process after $\mathrm{ICH}$, a number of preclinical and clinical studies regard antioxidants as the therapeutic strategies of hemorrhagic stroke [1,37]. Although plenty of preclinical studies exhibit good neuroprotection via scavenging ROS, there are still no effective drugs in the clinical treatment of ICH. Factors such as poor bloodbrain barrier (BBB) permeability and inefficient free radical scavenging capacity may contribute to treatment failure [37]. CeNPs are known to possess potent-free radical scavenging activity. CeNPs have both SOD mimetic (eradicating superoxide anions and hydroxyl radicals) and catalase mimetic (eradicating hydrogen peroxide), which enable it to eradicate ROS repetitively. Previous studies demonstrated that CeNP treatment could protect against BBB disruption and neuronal death in several CNS disorders [23, 38]. However, whether CeNP treatment will benefit WMI is still contentious. $\mathrm{ICH}$ commonly occurs around the basal ganglia, which contains abundant white matter fibers (internal capsule). It indicates that WMI is dominant after $\mathrm{ICH}$ instead of gray matter injury. Hence, the present study is designed to investigate the putative therapeutic effects of CeNP on white matter repair via its ROS scavenging capacity in regulating microglial and astrocyte cells.

As we know, ROS is highly elevated in perihematomal white matter after $\mathrm{ICH}$, which can oxidize protein, lipids, and nuclear material [15]. Oligodendrocyte-lineage cells (including OPCs and OLs) incorporate abundant lipids and lack essential glutathione and glutathione peroxidase. These specific characteristics make it highly vulnerable to oxidative stress and ultimately results in WMI [16]. The present study showed that ICH induced severe WMI, and PEG-CeNP treatment strongly reduced ROS levels in the brain tissue. In addition, PEG-CeNP treatment also improved the anatomical integrity of myelinated fibers and improved white matter integrity after ICH.

Notably, ROS plays a prominent role in microglia activation and M1/M2 shift [17-19]. As the resident immune cell in the CNS, substantial evidence supports the crucial role of microglia in coordinating oligodendroglia lineage cell responses during remyelination. M2 microglia promote OPC proliferation and differentiation by 
releasing neurotrophic factors (IGF-1, VEGF, IL-13), and also strengthen the phagocytosis of myelin debris [11, 39]. In contrast, M1 microglia favor the production and release of cytokines that aggravate OPCs and OLs damage [40]. Hence, manipulation of microglia polarization can be an effective strategy to promote remyelination (Fig. 9). PEG-CeNP treatment likely promotes remyelination in a microglia-dependent manner.

Our data showed that ICH strongly induced microglia M1 polarization, which may further hinder OPC differentiation and cause demyelination. Bosetti et al. demonstrated that microglial ROS scavenging through genetic abrogation of NADPH oxidase attenuates M1 response and elevates M2 markers [19]. Consistent with the previous study, the results of the RT-PCR, immunofluorescence, and flow cytometry study revealed that PEGCeNP treatment inhibited M1 polarization and promoted microglia toward M2 state.

As a prominent transcription factor, NF- $\mathrm{kB}$ is considered as a master inflammatory regulator, which is rapidly activated in the perihematomal region after $\mathrm{ICH}$ induction [15]. The NF- $\mathrm{kB}$ is a homo or heterodimer of the Rel family including 5 structural homologs: NF-kB1 (p50), NF-кB2 (p52), RelA (p65), RelB, and c-Rel. The p65/p50 dimer is the best-known inducer of an inflammatory response, which is fully functional in microglia [31]. Under normal conditions, ІкB- $\alpha$ always binds with the p65/p50 dimer to inhibit its activity [18]. ROS induce the inactivation and degradation of IkB- $\alpha$, which result in the translocation of p65/p50 dimer and then induce M1-polarized reaction [11, 31]. In the present study, we found that ICH strongly induced NF- $\mathrm{KB}$ p65 activation, while PEG-CeNP treatment reversed this. The results of western blot and immunofluorescence revealed that PEG-CeNP treatment hindered IкB- $\alpha$ degradation and the subsequent translocation of NF- $\mathrm{kB}$ p65 from the cytoplasm to the nucleus in microglia in vitro.

These results partly support our conjecture that PEGCeNP treatment promoted remyelination via inhibiting ROS-induced NF- $\mathrm{kB}$ p65 translocation and modulating skewed microglia polarization. Afterward, an inhibitor of colony-stimulating factor 1 receptor-PLX3397 was administrated for microglia depletion. We found that PEGCeNP treatment significantly enhanced the quantity of mature OLs, and such positive effects were substantially blocked after microglia depletion. Interestingly, we found that the number of mature OLs was slightly higher in $\mathrm{ICH}+\mathrm{CeNP}+$ PLX3397-treated mice than in those treated with $\mathrm{ICH}+$ vehicle + PLX3397. Therefore, we considered the possibility that astrocytes participated in the aforementioned pathophysiological process.

It is known that astrocytes play a critical role in remyelination [14, 34]. Their basic functions are essential in maintaining the extracellular matrix where oligodendrocytes survive and remyelinate the CNS. However, astrocytes can be detrimental under some pathological conditions, including neuroinflammation and oxidative stress [20, 34]. Barres et al. revealed that neuroinflammation and ischemia resulted in 2 different phenotypes of reactive astrocytes, termed $\mathrm{A} 1$ and $\mathrm{A} 2$ astrocytes [14]. The A1 neurotoxic astrocytes lose normal astrocytic functions and induce the death of neurons, OLs, and OPCs. A study on multiple sclerosis also demonstrated that $\mathrm{A} 1$ reactive astrocytes aggravated demyelination via hindering OPC differentiation and damaging
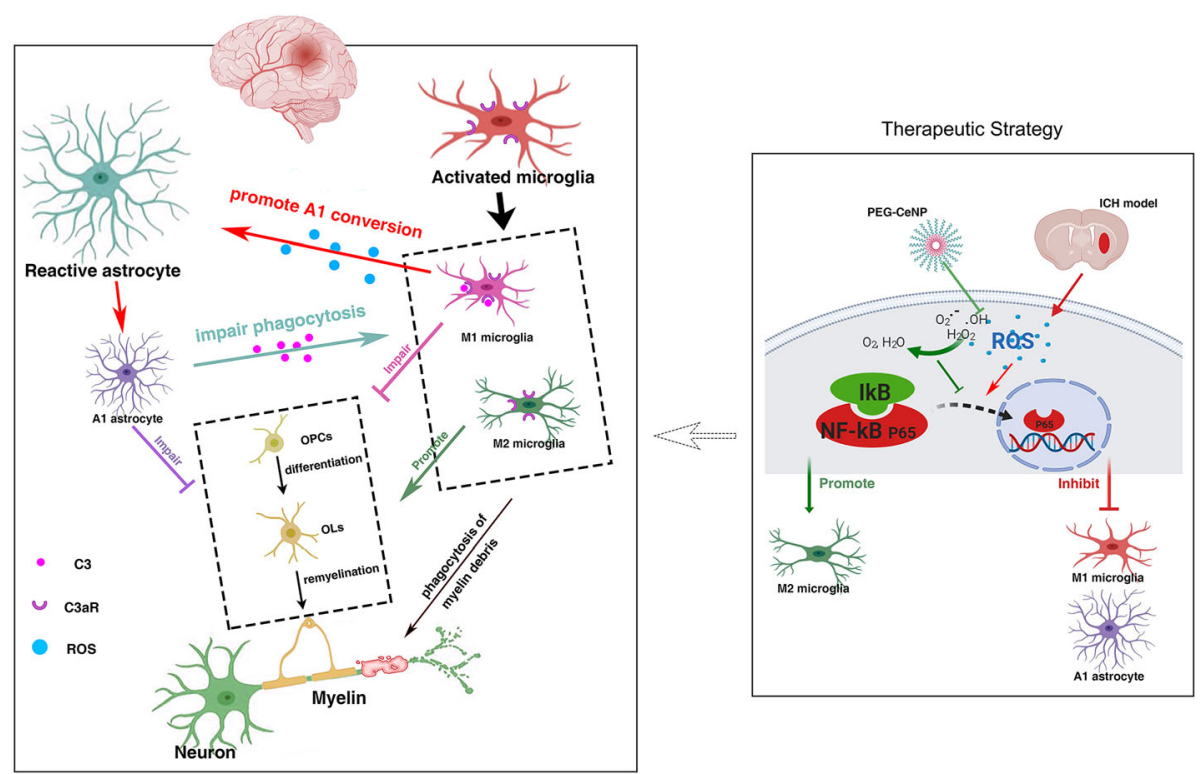

Fig. 9 Possible pathogenesis and therapeutic strategy 
differentiated OLs [28]. The RT-PCR results in our study showed that $\mathrm{C} 3$ mRNA expression at 7 days after $\mathrm{ICH}$ was approximately 200 folds higher than in the sham group. The immunostaining images of $\mathrm{C} 3$ and GFAP were also in accordance with the tendency of the $\mathrm{C} 3$ mRNA expression. These results showed that $\mathrm{ICH}$ could strongly induce A1 neurotoxic astrocytes.

Notably, A1 reactive astrocytes might be strongly associated with NF- $\mathrm{kB}$ activation [14, 34]. Recent studies have shown that amyloid- $\beta$ (A $\beta$ ) directly activated astrocytic NF- $\mathrm{kB}$ and $\mathrm{C} 3$ release, consistent with the high expression of $\mathrm{C} 3$ seen in patients with Alzheimer's disease [33]. Moreover, the $A \beta$-activated microglia $C M$ also induced A1 harmful astrocytes via the NF- $\mathrm{KB}$ signaling pathway [27]. In addition, ROS is known to be implicated in driving astrocyte reactivity and regulating NF$\kappa \mathrm{B}$ activation [20, 21]. In light of these results, we assumed that ROS might directly induce A1 astrocyte alteration via the NF- $\mathrm{kB}$ signaling pathway after $\mathrm{ICH}$. Then, A1 astrocyte exhibits detrimental effects on OPC differentiation and maturation (Fig. 9). The present study displayed that PEG-CeNP treatment resulted in the downregulation of $\mathrm{A} 1$ astrocyte expression after ICH. $\mathrm{H}_{2} \mathrm{O}_{2}$ treatment in vitro caused prominent translocation of NF- $\mathrm{KB}$ into the nucleus and increased $\mathrm{C} 3$ mRNA expression. PEG-CeNP treatment hindered A1 astrocyte expression, both in vivo and in vitro. These results help to verify our conjectures.

Except for the enhancement of OPC proliferation and differentiation, the clearance of myelin debris is also critical for white matter repair. Myelin debris hinders new myelin sheath extension and the recruitment/differentiation of oligodendrocyte-lineage cells. Microglia has the potent phagocytic potential for the engulfment of myelin debris, which is also essential for remyelination. In this study, we first reported that plenty of activated microglia were embedded in the astrocyte scar and exerted phagocytic capacity to remove debris after ICH. Notably, the complement cascade plays a crucial role in promoting synapse elimination by microglia [13]. C3/C3aR signaling is implicated in mediating microglial engulfment of redundant spines to ensure synaptic refinement under the physiological condition [29, 36]. The present study has displayed that A1 astrocytes highly expressed C3 both in vivo and in vitro. Meanwhile, C3aR was also abundantly expressed in microglia. A1 astrocytes may likely participate in mediating microglial engulfment of myelin debris via an astrocytic C3-microglial C3aR axis. As expected, the in vitro study indicated $\mathrm{C} 3$ and $\mathrm{A} 1$ astrocyte $\mathrm{CM}$ treatment strongly diminished fluorescent bead uptake. The negative effects of C3 and A1 astrocyte $\mathrm{CM}$ in microglial phagocytosis were substantially reversed after $\mathrm{C} 3 \mathrm{aR}$ antagonist administration. The electron micrograph also indicated that microglia exerted their phagocytic capacity to remove myelin debris in perihematomal region. Namely, astrocytes could decrease microglial engulfment of myelin debris via an astrocytic C3-microglial C3aR axis after ICH (Fig. 9). Therefore, it is of great value to inhibit A1 astrocyte alteration in the therapeutic strategy for $\mathrm{ICH}$.

Astrocytes and microglia have a unique bond and always respond as one unit when the brain is perturbed [13]. Microglia-astrocyte crosstalk is of burgeoning interest in numerous studies of CNS diseases. Recent work has illuminated that microglia induce A1 reactive astrocyte via secreting IL-1 $\alpha$, C1q, TNF after lipopolysaccharide stimulation in the CNS [14]. Moreover, astrocytederived IL-33 is implicated in regulating microgliaassociated synapse pruning and increasing chemokine expression in microglia $[13,41]$. In light of these previous studies, the results mentioned above in our study further replenish the feedback mechanism from astrocytes to microglia.

However, there remain some conjectures that have yet to be verified. As mentioned earlier, oligodendrocytelineage cells are rich in lipids, making them highly vulnerable to oxidative stress. The present study does not explore whether PEG-CeNP treatment directly promotes oligodendrocyte-lineage cell survival by eradicating intracellular ROS. In addition, the activated microglia are featured in producing and releasing abundant ROS to kill bacteria or cause neurotoxicity [42]. Since the present study has proved that ROS can directly induce A1 astrocyte, microglia may likely exacerbate A1 astrocyte alteration via releasing ROS (Fig. 9). Moreover, the expansion of the hematoma area can lead to secondary brain damage such as oxidative stress and inflammation and so on. The present study does not verify whether PEG-CeNP treatment can attenuate hematoma size. It is crucial and meaningful to investigate the relationship between microglia and hematoma. It is worth noting that astrocytes also play a vital role in the phagocytosis of synapses and clearance of debris. In diseases with severe myelin injury, including multiple sclerosis, and subacute ischemia, myelin debris is taken up by astrocytes through receptor-mediated endocytosis [43]. Hence, the role of astrocyte in the clearance of myelin debris and hematoma after $\mathrm{ICH}$ should be investigated in future studies.

\section{Conclusion}

In summary, the present study has uncovered a new mechanism in WMI after ICH. Namely, ICH induces M1 microglia and A1 astrocyte through ROS-induced NF- $\kappa B$ p65 translocation that hinders OPC differentiation and maturation. Subsequently, A1 astrocytes inhibit microglial engulfment of myelin debris via an astrocytic C3-microglial $\mathrm{C} 3 \mathrm{aR}$ axis. PEG-CeNP 
treatment inhibits ROS-induced NF-kB p65 translocation in both microglia and astrocytes and ultimately promotes remyelination. Such findings unveil a novel therapeutic strategy for white matter repair after $\mathrm{ICH}$. Future studies should regard astrocytes and microglia as a functional unit that has the potential to handle the complicated biological processes in new ways.

\section{Supplementary Information}

The online version contains supplementary material available at https://doi. org/10.1186/s12974-021-02101-6.

Additional file 1: Figure S1. Sample size and grouping information. Figure S2. Colloidal stability of PEG-CeNPs and normal CeNPs. Figure S3. Representative images of CD1632//ba1 and CD206//ba1 double immunostaining at 7 days post ICH. Lens: 200x; Scale bar: 100 um. Figure S4. Representative images of CD1632/lba1 and CD206//ba1 double immunostaining at 21 days post ICH. Lens: 200x; Scale bar: $100 \mu \mathrm{m}$. Figure S5. Representative images of olig2+ cells and EdU+ cells at 7 days post $\mathrm{ICH} .{ }^{*} \mathrm{p}<0.01$ versus $\mathrm{ICH}+$ vehicle, \# $\mathrm{p}<0.01$ versus $\mathrm{ICH}+\mathrm{CeNP}$, Lens: 200x; Scale bar: $100 \mu \mathrm{m}$. Figure S6. Representative images of GFAP and C3 double immunostaining in brain sections at 7 days post ICH. Lens: 200x; Scale bar: $100 \mu \mathrm{m}$. Figure S7. Representative images of GFAP and C3 double immunostaining in cultured astrocytes (control group). Lens: 200x; Scale bar: 50 um. Figure S8. Representative images of NF-kB p65 and GFAP double immunostaining in cultured astrocytes (control group). Lens: 400x; Scale bar: 25 m. Figure S9. Representative images of dMBP immunostaining at 3 days post $\mathrm{ICH}$. \# $p<0.05$ versus $\mathrm{ICH}+$ vehicle. Lens: 100x, 200x; Scale bar: $200 \mu \mathrm{m}$ (yellow), $100 \mu \mathrm{m}$ (white).

\section{Abbreviations}

BBB: Blood-brain barrier; CeNP: Ceria nanoparticles; CNS: Central nervous system; CM: Conditioned medium; C3: Complement component 3; C3aR: C3a receptor; C3aRA: C3aR antagonist; DHE: Dihydroethidium; ICH: Intracerebral hemorrhage; OPCs: Oligodendrocyte progenitor cells; OLs: Oligodendrocytes; PEG-CeNPs: Polyethylene glycol-CeNPs; PDL: Poly-D-lysine;

PVDF: Polyvinylidene fluoride; ROS: Reactive oxygen species; SOD: Superoxide dismutase; SEM: Standard error of the mean; TEM: Transmission electron microscopy

\section{Acknowledgements}

The BioRender software was used to draw the schematic diagram.

\section{Authors' contributions}

$J M Z, F L$ and JY are the principal investigators, they design the study. JWZ, JNL, SHM and HJW contribute to the study design, experiment implementation and manuscript draft. ZYS, YJF, SBX and XYW summarize and analyze the experimental data and make suggestions to the improvement of the study. LGS, WLX and SC revised the manuscript and polish the language. The author(s) read and approved the final manuscript.

\section{Funding}

This study was supported by the Key Program of Science and Technology Development of Zhejiang Province (2017C03021) and National Natural Science Foundation of China (81870916) to Jianmin Zhang; National Natural Science Foundation of China (81901676) to Feng Liang; National Natural Science Foundation of China (81971097) to Jun Yu; National Natural Science Foundation of China (81701214) to Haijian Wu.

\section{Availability of data and materials}

All raw data used in this manuscript are available on reasonable request

\section{Ethics approval and consent to participate}

Not applicable

\section{Consent for publication}

Not applicable

\section{Competing interests}

The authors declare no conflict of interest.

\section{Author details}

${ }^{1}$ Department of Neurosurgery, Second Affiliated Hospital, School of Medicine, Zhejiang University, Hangzhou, Zhejiang, China. ${ }^{2}$ Brain Research Institute, Zhejiang University, Hangzhou, Zhejiang, China. ${ }^{3}$ Collaborative Innovation Center for Brain Science, Zhejiang University, Hangzhou, Zhejiang, China.

Received: 29 September 2020 Accepted: 3 February 2021

Published online: 15 February 2021

\section{References}

1. Keep RF, Hua Y, Xi G. Intracerebral haemorrhage: mechanisms of injury and therapeutic targets. Lancet Neurol. 2012;11(8):720-31.

2. Zheng J, Sun Z, Liang F, Xu W, Lu J, Shi L, et al. AdipoRon attenuates neuroinflammation after intracerebral hemorrhage through AdipoR1-AMPK pathway. Neuroscience. 2019;412:116-30.

3. Zhang Q, Zhu W, Xu F, Dai X, Shi L, Cai W, et al. The interleukin-4/ PPARgamma signaling axis promotes oligodendrocyte differentiation and remyelination after brain injury. PLoS Biol. 2019;17(6):e3000330.

4. Jiang $Y B$, Wei KY, Zhang $X Y$, Feng $H$, Hu R. White matter repair and treatment strategy after intracerebral hemorrhage. CNS Neurosci Ther. 2019; 25(10):1113-25.

5. Smith EE, Gurol ME, Eng JA, Engel CR, Nguyen TN, Rosand J, et al. White matter lesions, cognition, and recurrent hemorrhage in lobar intracerebral hemorrhage. Neurology. 2004;63(9):1606-12.

6. McKenzie IA, Ohayon D, Li H, de Faria JP, Emery B, Tohyama K, et al. Motor skill learning requires active central myelination. Science. 2014;346(6207): 318-22.

7. Mekhail M, Almazan G, Tabrizian M. Oligodendrocyte-protection and remyelination post-spinal cord injuries: a review. Prog Neurobiol. 2012;96(3): 322-39.

8. Kang M, Yao Y. Oligodendrocytes in intracerebral hemorrhage. CNS Neurosci Ther. 2019:25(10):1075-84.

9. Franklin RJ, Ffrench-Constant C. Remyelination in the CNS: from biology to therapy. Nat Rev Neurosci. 2008;9(11):839-55.

10. Joseph MJ, Caliaperumal J, Schlichter LC. After intracerebral hemorrhage, oligodendrocyte precursors proliferate and differentiate inside white-matter tracts in the rat striatum. Transl Stroke Res. 2016;7(3):192-208.

11. Lan X, Han X, Li Q, Yang QW, Wang J. Modulators of microglial activation and polarization after intracerebral haemorrhage. Nat Rev Neurol. 2017;13(7): 420-33.

12. Miron VE, Boyd A, Zhao JW, Yuen TJ, Ruckh JM, Shadrach JL, et al. M2 microglia and macrophages drive oligodendrocyte differentiation during CNS remyelination. Nat Neurosci. 2013;16(9):1211-8.

13. Vainchtein ID, Molofsky AV. Astrocytes and microglia: in sickness and in health. Trends Neurosci. 2020:43(3):144-54.

14. Liddelow SA, Guttenplan KA, Clarke LE, Bennett FC, Bohlen CJ, Schirmer L, et al. Neurotoxic reactive astrocytes are induced by activated microglia. Nature. 2017:541(7638):481-7.

15. Wagner KR. Modeling intracerebal hemorrhage - Glutamate, nuclear factorkappa B signaling and cytokines. Stroke. 2007;38(2):753-8.

16. Bagi Z, Brandner DD, Le P, McNeal DW, Gong X, Dou H, et al. Vasodilator dysfunction and oligodendrocyte dysmaturation in aging white matter. Ann Neurol. 2018;83(1):142-52.

17. Taetzsch T, Levesque S, McGraw C, Brookins S, Luqa R, Bonini MG, et al. Redox regulation of NF-kappaB p50 and M1 polarization in microglia. Glia. 2015:63(3):423-40

18. Zeng F, Wu YW, Li XW, Ge XJ, Guo QH, Lou XB, et al. Custom-made ceria nanoparticles show a neuroprotective effect by modulating phenotypic polarization of the microglia. Angew Chem Int Edit. 2018;57(20):5808-12.

19. Choi SH, Aid S, Kim HW, Jackson SH, Bosetti F. Inhibition of NADPH oxidase promotes alternative and anti-inflammatory microglial activation during neuroinflammation. J Neurochem. 2012;120(2):292-301.

20. Verkhratsky A, Steardo L, Parpura V, Montana V. Translational potential of astrocytes in brain disorders. Prog Neurobiol. 2016;144:188-205.

21. Xian $P$, Hei $Y$, Wang R, Wang T, Yang J, Li J, et al. Mesenchymal stem cellderived exosomes as a nanotherapeutic agent for amelioration of inflammation-induced astrocyte alterations in mice. Theranostics. 2019:9(20): 5956-75. 
22. Ganesana M, Erlichman JS, Andreescu S. Real-time monitoring of superoxide accumulation and antioxidant activity in a brain slice model using an electrochemical cytochrome c biosensor. Free Radical Bio Med. 2012;53(12): 2240-9.

23. Bao Q, Hu P, Xu Y, Cheng T, Wei C, Pan L, et al. Simultaneous blood-brain barrier crossing and protection for stroke treatment based on edaravoneloaded ceria nanoparticles. ACS Nano. 2018;12(7):6794-805.

24. Elmore MRP, Najafi AR, Koike MA, Dagher NN, Spangenberg EE, Rice RA, et al. Colony-stimulating factor 1 receptor signaling is necessary for microglia viability, unmasking a microglia progenitor cell in the adult brain. Neuron. 2014;82(2):380-97.

25. Rodriguez C, Sobrino T, Agulla J, Bobo-Jimenez V, Ramos-Araque ME, Duarte $\mathrm{J}$, et al. Neovascularization and functional recovery after intracerebral hemorrhage is conditioned by the Tp53 Arg72Pro single-nucleotide polymorphism. Cell Death Differ. 2017;24(1):144-54.

26. Kim D, You B, Jo EK, Han SK, Simon MI, Lee SJ. NADPH oxidase 2-derived reactive oxygen species in spinal cord microglia contribute to peripheral nerve injury-induced neuropathic pain. Proc Natl Acad Sci U S A. 2010; 107(33):14851-6.

27. Xu XT, Zhang AW, Zhu YT, He W, Di W, Fang YN, et al. MFG-E8 reverses microglial-induced neurotoxic astrocyte (A1) via NF-kappa B and PI3K-Akt pathways. J Cell Physiol. 2019;234(1):904-14.

28. Lombardi M, Parolisi R, Scaroni F, Bonfanti E, Gualerzi A, Gabrielli M, et al. Detrimental and protective action of microglial extracellular vesicles on myelin lesions: astrocyte involvement in remyelination failure. Acta Neuropathol. 2019;138(6):987-1012.

29. Lian H, Litvinchuk A, Chiang ACA, Aithmitti N, Jankowsky JL, Zheng H. Astrocyte-microglia cross talk through complement activation modulates amyloid pathology in mouse models of Alzheimer's disease. J Neurosci. 2016;36(2):577-89.

30. Zeng CB, Pan FH, Jones LA, Lim MM, Griffin EA, Sheline Yl, et al. Evaluation of 5-ethynyl-2 '-deoxyuridine staining as a sensitive and reliable method for studying cell proliferation in the adult nervous system. Brain Res. 2010;1319: 21-32.

31. Rojo Al, McBean G, Cindric M, Egea J, Lopez MG, Rada P, et al. Redox control of microglial function: molecular mechanisms and functional significance. Antioxid Redox Signal. 2014;21(12):1766-801.

32. Peferoen L, Kipp M, van der Valk P, van Noort JM, Amor S. Oligodendrocytemicroglia cross-talk in the central nervous system. Immunology. 2014;141(3): 302-13

33. Lian H, Yang L, Cole A, Sun L, Chiang AC, Fowler SW, et al. NFkappaBactivated astroglial release of complement C3 compromises neuronal morphology and function associated with Alzheimer's disease. Neuron. 2015:85(1):101-15.

34. Liddelow SA, Barres BA. Reactive astrocytes: production, function, and therapeutic potential. Immunity. 2017;46(6):957-67.

35. Silver J, Miller JH. Regeneration beyond the glial scar. Nature Reviews Neuroscience. 2004;5(2):146-56.

36. Stevens B, Allen NJ, Vazquez LE, Howell GR, Christopherson KS, Nouri N, et al. The classical complement cascade mediates CNS synapse elimination. Cell. 2007;131(6):1164-78.

37. Aronowski J, Zhao X. Molecular pathophysiology of cerebral hemorrhage: secondary brain injury. Stroke. 2011;42(6):1781-6.

38. Jeong HG, Cha BG, Kang DW, Kim DY, Ki SK, Kim SI, et al. Ceria Nanoparticles synthesized with aminocaproic acid for the treatment of subarachnoid hemorrhage. Stroke. 2018;49(12):3030-8.

39. Kotter MR, Li WW, Zhao C, Franklin RJ. Myelin impairs CNS remyelination by inhibiting oligodendrocyte precursor cell differentiation. J Neurosci. 2006; 26(1):328-32.

40. Hu X, Leak RK, Shi Y, Suenaga J, Gao Y, Zheng P, et al. Microglial and macrophage polarization-new prospects for brain repair. Nat Rev Neurol. 2015;11(1):56-64.

41. Vainchtein ID, Chin G, Cho FS, Kelley KW, Miller JG, Chien EC, et al. Astrocyte-derived interleukin-33 promotes microglial synapse engulfment and neural circuit development. Science. 2018;359(6381):1269-73.

42. Block ML, Zecca L, Hong JS. Microglia-mediated neurotoxicity: uncovering the molecular mechanisms. Nat Rev Neurosci. 2007;8(1):57-69.

43. Ponath G, Ramanan S, Mubarak M, Housley W, Lee S, Sahinkaya FR, et al. Myelin phagocytosis by astrocytes after myelin damage promotes lesion pathology. Brain. 2017;140(2):399-413.

\section{Publisher's Note}

Springer Nature remains neutral with regard to jurisdictional claims in published maps and institutional affiliations.
Ready to submit your research? Choose BMC and benefit from:

- fast, convenient online submission

- thorough peer review by experienced researchers in your field

- rapid publication on acceptance

- support for research data, including large and complex data types

- gold Open Access which fosters wider collaboration and increased citations

- maximum visibility for your research: over $100 \mathrm{M}$ website views per year

At BMC, research is always in progress.

Learn more biomedcentral.com/submissions 